\title{
GL(3)-Based Quantum Integrable Composite Models. II. Form Factors of Local Operators
}

\author{
Stanislav PAKULIAK ${ }^{a b c}$, Eric RAGOUCY ${ }^{d}$ and Nikita A. SLAVNOV ${ }^{e}$ \\ ${ }^{a}$ Institute of Theoretical \&5 Experimental Physics, 117259 Moscow, Russia \\ ${ }^{b}$ Laboratory of Theoretical Physics, JINR, 141980 Dubna, Moscow reg., Russia \\ E-mail: pakuliak@jinr.ru \\ ${ }^{c}$ Moscow Institute of Physics and Technology, 141700, Dolgoprudny, Moscow reg., Russia \\ ${ }^{d}$ Laboratoire de Physique Théorique LAPTH, CNRS and Université de Savoie, \\ BP 110, 74941 Annecy-le-Vieux Cedex, France \\ E-mail: eric.ragoucy@lapth.cnrs.fr \\ e Steklov Mathematical Institute, Moscow, Russia \\ E-mail: nslavnov@mi.ras.ru
}

Received February 18, 2015, in final form July 22, 2015; Published online July 31, 2015

http://dx.doi.org/10.3842/SIGMA.2015.064

\begin{abstract}
We study integrable models solvable by the nested algebraic Bethe ansatz and possessing the GL(3)-invariant $R$-matrix. We consider a composite model where the total monodromy matrix of the model is presented as a product of two partial monodromy matrices. Assuming that the last ones can be expanded into series with respect to the inverse spectral parameter we calculate matrix elements of the local operators in the basis of the transfer matrix eigenstates. We obtain determinant representations for these matrix elements. Thus, we solve the inverse scattering problem in a weak sense.
\end{abstract}

Key words: Bethe ansatz; quantum affine algebras, composite models

2010 Mathematics Subject Classification: 17B37; 81R50

\section{Introduction}

The algebraic Bethe ansatz was found to be a powerful method for describing the spectrum of various quantum integrable models $[7,23,33,36]$. In this approach, quantum Hamiltonians and all other integrals of motion are generated by a transfer matrix. The eigenstates of the latest can be found in a systematic way, leading to a set of equations determining the spectrum (Bethe equations).

Despite the significant progress of the algebraic Bethe ansatz in calculating the spectrum, the application of this technique to the problem of calculating correlation functions for a long time led to much more limited results. It is worth mentioning the papers $[10,11,21]$, where series representations for correlations of the model of one-dimensional bosons were obtained. Later, Fredholm determinant representations for correlation functions of this model were derived by the method of dual fields (see [22] and references therein).

Solution of the quantum inverse scattering problem [20,25] has opened up new opportunities of the algebraic Bethe ansatz. Using this result correlation functions of the $X X Z$ spin chain were studied in series of works $[8,9,15,18,19]$. The explicit formulas for the local operators provided by the inverse scattering problem also had played an important role in calculating their form factors $[5,16,17]$. It is worth mentioning, however, that the results of $[20,25]$ essentially were based on the fact that the monodromy matrix of the model could be constructed from the 
$R$-matrix. This is true for various spin chains, but not in general. In the present paper we use the approach of [10] for calculating form factors of local operators in quantum GL(3)-invariant models. Let us briefly describe the main idea of this method.

The key equation of the quantum inverse scattering method is the $R T T$-relation $[33,36]$

$$
R_{12}(u, v) T_{1}(u) T_{2}(v)=T_{2}(v) T_{1}(u) R_{12}(u, v) .
$$

Here $T(u)$ is the monodromy matrix, $R(u, v)$ is the $R$-matrix. In GL(3)-invariant models the $R$-matrix acts in the tensor product of two auxiliary spaces $V_{1} \otimes V_{2}\left(V_{k} \sim \mathbb{C}^{3}, k=1,2\right)$ and has the form

$$
R(u, v)=\mathbf{I}+g(u, v) \mathbf{P}, \quad g(u, v)=\frac{c}{u-v} .
$$

Here $\mathbf{I}$ is the identity matrix in $V_{1} \otimes V_{2}, \mathbf{P}$ is the permutation matrix that exchanges $V_{1}$ and $V_{2}$, and $c$ is a constant. The monodromy matrix $T(u)$ acts in $\mathbb{C}^{3} \otimes \mathcal{H}$, where $\mathcal{H}$ is the Hilbert space of the Hamiltonian of the model under consideration. Equation (1.1) holds in the tensor product $V_{1} \otimes V_{2} \otimes \mathcal{H}$, and the matrices $T_{k}(w)$ act non-trivially in $V_{k} \otimes \mathcal{H}$.

The monodromy matrix $T(u)$ of a lattice quantum model is equal to the product of local $L$-operators

$$
T(u)=L_{M}(u) \cdots L_{1}(u),
$$

where $M$ is the number of lattice sites, and every $L$-operator satisfies the $R T T$-relation with the $R$-matrix (1.2). Continuous quantum models appear in the limit $M \rightarrow \infty$. Let us fix some site $m(1 \leq m<M)$ and define two partial monodromy matrices $T^{(1)}(u)$ and $T^{(2)}(u)$ as

$$
T^{(1)}(u)=L_{m}(u) \cdots L_{1}(u), \quad T^{(2)}(u)=L_{M}(u) \cdots L_{m+1}(u) .
$$

Then obviously

$$
T(u)=T^{(2)}(u) T^{(1)}(u) .
$$

We call such model composite generalized model ${ }^{1}[10]$.

We assume that local $L$-operators in (1.3) depend on the spectral parameter $u$ as follows ${ }^{2}$ :

$$
L_{n}(u)=\mathbf{1}+\frac{c}{u} L_{n}[0]+o\left(u^{-1}\right), \quad u \rightarrow \infty .
$$

Here 1 is the identity operator in $\mathbb{C}^{3} \otimes \mathcal{H}$, and the matrix elements of $L_{n}[0]$ are local operators of the model. Then it is easy to see that both partial monodromy matrices $T^{(l)}(u)$ have the standard expansion over $c / u$ :

$$
T^{(l)}(u)=\mathbf{1}+\sum_{n=0}^{\infty} T^{(l)}[n]\left(\frac{c}{u}\right)^{n+1}, \quad l=1,2,
$$

where the partial zero mode $T^{(1)}[0]$ is equal to

$$
T^{(1)}[0]=\sum_{n=1}^{m} L_{n}[0] .
$$

\footnotetext{
${ }^{1}$ The authors of [10] used the terminology two-site model. We think that this terminology becomes misleading in the case of spin chains. The terminology two-component model used in [34] also becomes misleading in the case of multi-component Bose or Fermi gases. We choose to use the terminology introduced in [29].

${ }^{2}$ See [24] for concrete examples of $L$-operators satisfying the $R T T$-relation with the GL(3)-invariant $R$-matrix.
} 
In this paper we develop a method of calculating form factors of matrix elements $T_{i j}^{(1)}[0]$. We reduce them to the form factors of the monodromy matrix entries $T_{i j}(z)$ studied in our previous publications. In this way we obtain determinant representations for the form factors of $T_{i j}^{(1)}[0]$ and extract explicitly their dependence on the lattice site number $m$. Then, taking the lattice derivative of the results obtained, we find determinant representations for the form factors of the local operators $\left(L_{m}[0]\right)_{i j}$. We do not give here explicit determinant formulas, but the reader can find them in $[2,4,27,28,30]$.

The paper is organized as follows. In Section 2 we introduce basic notions of the generalized model. In Section 3 we consider composite generalized model. Section 4 contains the main results of this paper. There we reduce form factors of local operators to the ones of the monodromy matrix entries for the GL(3) case and we conjecture a form for the GL $(N)$ case. The following sections contain the proofs for GL(3). In Section 5 we consider the form factors of the diagonal partial zero modes $T_{i i}^{(1)}[0]$. Finally, in Section 6 we study the form factors of the partial zero $\operatorname{modes} T_{i j}^{(1)}[0]$ for $i \neq j$.

\section{Generalized model}

\subsection{Bethe vectors}

In the framework of the algebraic Bethe ansatz the entries of $T(w)$ act in a Hilbert space $\mathcal{H}$ and its dual $\mathcal{H}^{*}$ that possess a pseudovacuum vector $|0\rangle$ and a dual pseudovacuum vector $\langle 0|$ respectively. They are normalized by the condition $\langle 0 \mid 0\rangle=1$. These vectors are annihilated by the operators $T_{i j}(w)$, where $i>j$ for $|0\rangle$ and $i<j$ for $\langle 0|$. At the same time both vectors are eigenvectors for the diagonal entries of the monodromy matrix

$$
T_{i i}(w)|0\rangle=\lambda_{i}(w)|0\rangle, \quad\langle 0| T_{i i}(w)=\lambda_{i}(w)\langle 0|, \quad i=1,2,3,
$$

where $\lambda_{i}(w)$ are some scalar functions. In the framework of the generalized model, $\lambda_{i}(w)$ remain free functional parameters. Actually, it is always possible to normalize the monodromy matrix $T(w) \rightarrow \lambda_{2}^{-1}(w) T(w)$ so as to deal only with the ratios

$$
r_{1}(w)=\frac{\lambda_{1}(w)}{\lambda_{2}(w)}, \quad r_{3}(w)=\frac{\lambda_{3}(w)}{\lambda_{2}(w)} .
$$

Below we assume that $\lambda_{2}(w)=1$.

Bethe vectors are certain polynomials in the operators $T_{i j}(u)$ with $i<j$ acting on the pseudovacuum vector $|0\rangle[3,12,13,23,37]$. In the GL(3)-invariant models they depend on two sets of variables called Bethe parameters. We denote the Bethe vectors $\mathbb{B}_{a, b}(\bar{u} ; \bar{v})$. Here the Bethe parameters are $\bar{u}=\left\{u_{1}, \ldots, u_{a}\right\}$ and $\bar{v}=\left\{v_{1}, \ldots, v_{b}\right\}$. The subscripts $a$ and $b(a, b=0,1, \ldots)$ respectively denote the cardinalities of the sets $\bar{u}$ and $\bar{v}$.

Similarly we can construct dual Bethe vectors in the dual space as polynomials in the operators $T_{i j}(u)$ with $i>j$ acting on the dual pseudovacuum vector $\langle 0|$. We denote them $\mathbb{C}_{a, b}(\bar{u} ; \bar{v})$ with the same meaning of the arguments and subscripts.

\subsection{Notation}

Besides the function $g(u, v)$ we also introduce a function $f(u, v)$

$$
f(u, v)=\frac{u-v+c}{u-v} .
$$

We denote sets of variables by bar: $\bar{u}, \bar{v}$ etc. If necessary, the cardinalities of the sets are given in special comments. Individual elements of the sets are denoted by subscripts: $w_{j}, u_{k}$ 
etc. We say that $\bar{x}=\bar{x}^{\prime}$, if $\# \bar{x}=\# \bar{x}^{\prime}$ and $x_{i}=x_{i}^{\prime}$ (up to a permutation) for $i=1, \ldots, \# \bar{x}$. We say that $\bar{x} \neq \bar{x}^{\prime}$ otherwise.

Below we consider partitions of sets into subsets. The notation $\bar{u} \Rightarrow\left\{\bar{u}_{\mathrm{I}}, \bar{u}_{\mathrm{II}}\right\}$ means that the set $\bar{u}$ is divided into two disjoint subsets. As a rule, we use roman numbers for subscripts of subsets: $\bar{u}_{\mathrm{I}}, \bar{v}_{\text {ii }}$ etc. However, if we deal with a big quantity of subsets, then we use standard arabic numbers for their notation. In such cases we give special comments to avoid ambiguities.

Similarly to our previous papers (see, for instance, [29]) we use a shorthand notation for products of some functions. Namely, if the functions $r_{k}(2.1)$ or the function $f(2.2)$ depend on sets of variables, this means that one should take the product over the corresponding set. For example,

$$
r_{1}(\bar{u})=\prod_{u_{k} \in \bar{u}} r_{1}\left(u_{k}\right), \quad f(z, \bar{w})=\prod_{w_{j} \in \bar{w}} f\left(z, w_{j}\right), \quad f(\bar{u}, \bar{v})=\prod_{u_{j} \in \bar{u}} \prod_{v_{k} \in \bar{v}} f\left(u_{j}, v_{k}\right) .
$$

By definition any product with respect to the empty set is equal to 1 . If we have a double product, then it is also equal to 1 if at least one of sets is empty.

In Section 3 we shall introduce several new scalar functions and will extend the convention (2.3) to their products.

\subsection{On-shell Bethe vectors}

In the algebraic Bethe ansatz the role of a quantum Hamiltonian is played by the transfer matrix. It is the trace in the auxiliary space of the monodromy matrix: $\operatorname{tr} T(u)$. The eigenstates of the transfer matrix are called on-shell Bethe vectors. The eigenstates of the transfer matrix in the dual space are called dual on-shell Bethe vectors ${ }^{3}$. We will denote usual and dual Bethe vectors as $\mathbb{B}_{a, b}(\bar{u}, \bar{v})$ and $\mathbb{C}_{a, b}(\bar{u}, \bar{v})$ respectively. Different equivalent formulas for these Bethe vectors were presented in the paper [3]. In what follows we will not use these explicit expressions. Instead, we will use formulas which relate Bethe vectors of the composite model with the ones for the components of the model (see formulas (3.4) and (3.6) which are proved in the first part of this paper [29]) and the action of the monodromy matrix elements onto Bethe vectors obtained in [3].

A (dual) Bethe vector becomes on-shell, if the Bethe parameters satisfy the system of Bethe equations. We give this system in a slightly unusual form

$$
r_{1}\left(\bar{u}_{\mathrm{I}}\right)=\frac{f\left(\bar{u}_{\mathrm{I}}, \bar{u}_{\mathrm{II}}\right)}{f\left(\bar{u}_{\mathrm{II}}, \bar{u}_{\mathrm{I}}\right)} f\left(\bar{v}, \bar{u}_{\mathrm{I}}\right), \quad r_{3}\left(\bar{v}_{\mathrm{I}}\right)=\frac{f\left(\bar{v}_{\mathrm{II}}, \bar{v}_{\mathrm{I}}\right)}{f\left(\bar{v}_{\mathrm{I}}, \bar{v}_{\mathrm{II}}\right)} f\left(\bar{v}_{\mathrm{I}}, \bar{u}\right) .
$$

These equations should hold for arbitrary partitions of the sets $\bar{u}$ and $\bar{v}$ into subsets $\left\{\bar{u}_{\mathrm{I}}, \bar{u}_{\mathrm{II}}\right\}$ and $\left\{\bar{v}_{\mathrm{I}}, \bar{v}_{\mathrm{II}}\right\}$ respectively. Obviously, it is enough to demand that the system (2.4) is valid for the particular case, when the sets $\bar{u}_{\mathrm{I}}$ and $\bar{v}_{\mathrm{I}}$ consist of only one element. Then it turns into the standard system of Bethe equations.

If the sets $\bar{u}$ and $\bar{v}$ satisfy (2.4), then

$$
\operatorname{tr} T(w) \mathbb{B}_{a, b}(\bar{u}, \bar{v})=\tau(w \mid \bar{u}, \bar{v}) \mathbb{B}_{a, b}(\bar{u}, \bar{v}), \quad \mathbb{C}_{a, b}(\bar{u}, \bar{v}) \operatorname{tr} T(w)=\tau(w \mid \bar{u}, \bar{v}) \mathbb{C}_{a, b}(\bar{u}, \bar{v}),
$$

with

$$
\tau(w \mid \bar{u}, \bar{v})=r_{1}(w) f(\bar{u}, w)+f(w, \bar{u}) f(\bar{v}, w)+r_{3}(w) f(w, \bar{v}) .
$$

Besides usual on-shell Bethe vectors it is also convenient to consider twisted on-shell Bethe vectors (see, e.g., [2]). They are eigenstates of a twisted transfer matrix, that in its turn, is the

\footnotetext{
${ }^{3}$ For simplicity here and below we do not distinguish between vectors and dual vectors, because their properties are completely analogous to each other.
} 
trace in the auxiliary space of the twisted monodromy matrix $T_{\bar{\kappa}}(u)$. The last one is defined as $T_{\bar{\kappa}}(u)=\hat{\kappa} T(u)$, where $\hat{\kappa}=\operatorname{diag}\left(\kappa_{1}, \kappa_{2}, \kappa_{3}\right)$. The matrix elements $\kappa_{i}(i=1,2,3)$ are called twist parameters. A (dual) Bethe vector becomes twisted on-shell vector, if the Bethe parameters satisfy the system of twisted Bethe equations:

$$
r_{1}\left(\bar{u}_{\mathrm{I}}\right)=\left(\frac{\kappa_{2}}{\kappa_{1}}\right)^{k_{\mathrm{I}}} \frac{f\left(\bar{u}_{\mathrm{I}}, \bar{u}_{\mathrm{II}}\right)}{f\left(\bar{u}_{\mathrm{II}}, \bar{u}_{\mathrm{I}}\right)} f\left(\bar{v}, \bar{u}_{\mathrm{I}}\right), \quad r_{3}\left(\bar{v}_{\mathrm{I}}\right)=\left(\frac{\kappa_{2}}{\kappa_{3}}\right)^{n_{\mathrm{I}}} \frac{f\left(\bar{v}_{\mathrm{II}}, \bar{v}_{\mathrm{I}}\right)}{f\left(\bar{v}_{\mathrm{I}}, \bar{v}_{\mathrm{II}}\right)} f\left(\bar{v}_{\mathrm{I}}, \bar{u}\right),
$$

where $k_{\mathrm{I}}=\# \bar{u}_{\mathrm{I}}$ and $n_{\mathrm{I}}=\# \bar{v}_{\mathrm{I}}$. These equations also should hold for arbitrary partitions of the sets $\bar{u}$ and $\bar{v}$ into subsets.

\subsection{Description of scalar products}

Recall a formula for the scalar product of generic Bethe vectors [32]:

$$
\begin{aligned}
\mathcal{S}_{a, b} \equiv & \mathbb{C}_{a, b}\left(\bar{u}^{C} ; \bar{v}^{C}\right) \mathbb{B}_{a, b}\left(\bar{u}^{B} ; \bar{v}^{B}\right)=\sum r_{1}\left(\bar{u}_{\mathrm{I}}^{B}\right) r_{1}\left(\bar{u}_{\mathrm{II}}^{C}\right) r_{3}\left(\bar{v}_{\mathrm{II}}^{C}\right) r_{3}\left(\bar{v}_{\mathrm{I}}^{B}\right) f\left(\bar{u}_{\mathrm{I}}^{C}, \bar{u}_{\mathrm{II}}^{C}\right) f\left(\bar{u}_{\mathrm{II}}^{B}, \bar{u}_{\mathrm{I}}^{B}\right) \\
& \times f\left(\bar{v}_{\mathrm{II}}^{C}, \bar{v}_{\mathrm{I}}^{C}\right) f\left(\bar{v}_{\mathrm{I}}^{B}, \bar{v}_{\mathrm{II}}^{B}\right) \frac{f\left(\bar{v}_{\mathrm{I}}^{C}, \bar{u}_{\mathrm{I}}^{C}\right) f\left(\bar{v}_{\mathrm{II}}^{B}, \bar{u}_{\mathrm{II}}^{B}\right)}{f\left(\bar{v}^{C}, \bar{u}^{C}\right) f\left(\bar{v}^{B}, \bar{u}^{B}\right)} Z_{a_{\mathrm{II}}, b_{\mathrm{I}}}\left(\bar{u}_{\mathrm{II}}^{C} ; \bar{u}_{\mathrm{II}}^{B} \mid \bar{v}_{\mathrm{I}}^{C} ; \bar{v}_{\mathrm{I}}^{B}\right) Z_{a_{\mathrm{I}}, b_{\mathrm{II}}}\left(\bar{u}_{\mathrm{I}}^{B} ; \bar{u}_{\mathrm{I}}^{C} \mid \bar{v}_{\mathrm{II}}^{B} ; \bar{v}_{\mathrm{II}}^{C}\right)
\end{aligned}
$$

Here all the Bethe parameters are generic complex numbers and the sum is taken over the partitions of the sets $\bar{u}^{C}, \bar{u}^{B}, \bar{v}^{C}$, and $\bar{v}^{B}$

$$
\begin{array}{ll}
\bar{u}^{C} \Rightarrow\left\{\bar{u}_{\mathrm{I}}^{C}, \bar{u}_{\mathrm{II}}^{C}\right\}, & \bar{v}^{C} \Rightarrow\left\{\bar{v}_{\mathrm{I}}^{C}, \bar{v}_{\mathrm{II}}^{C}\right\}, \\
\bar{u}^{B} \Rightarrow\left\{\bar{u}_{\mathrm{I}}^{B}, \bar{u}_{\mathrm{II}}^{B}\right\}, & \bar{v}^{B} \Rightarrow\left\{\bar{v}_{\mathrm{I}}^{B}, \bar{v}_{\mathrm{II}}^{B}\right\} .
\end{array}
$$

The partitions are independent except that $\# \bar{u}_{\mathrm{I}}^{B}=\# \bar{u}_{\mathrm{I}}^{C}=a_{\mathrm{I}}$ with $a_{\mathrm{I}}=0, \ldots, a$, and $\# \bar{v}_{\mathrm{I}}^{B}=$ $\# \bar{v}_{\mathrm{I}}^{C}=b_{\mathrm{I}}$ with $b_{\mathrm{I}}=0, \ldots, b$. From this we find $\# \bar{u}_{\mathrm{II}}^{B}=\# \bar{u}_{\mathrm{II}}^{C}=a_{\mathrm{II}}=a-a_{\mathrm{I}}$ and $\# \bar{v}_{\mathrm{II}}^{B}=\# \bar{v}_{\mathrm{II}}^{C}=$ $b_{\mathrm{II}}=b-b_{\mathrm{I}}$. The functions $Z_{a_{\mathrm{II}}, b_{\mathrm{I}}}$ and $Z_{a_{\mathrm{I}}, b_{\mathrm{II}}}$ are so-called highest coefficients. They are equal to a partition function of 15 -vertex model with special boundary conditions [32]. The reader can find their explicit representations in $[1,38]$. We do not use these explicit formulas in the present paper except $Z_{0,0}(\varnothing ; \varnothing \mid \varnothing ; \varnothing)=1$. This condition is needed to satisfy the normalization $\mathcal{S}_{0,0}=\langle 0 \mid 0\rangle=1$.

If $\mathbb{C}_{a, b}\left(\bar{u}^{C} ; \bar{v}^{C}\right)$ is a twisted on-shell vector and $\mathbb{B}_{a, b}\left(\bar{u}^{B} ; \bar{v}^{B}\right)$ is a usual on-shell vector, then we can express the functions $r_{1}\left(u_{j}^{C, B}\right)$ and $r_{3}\left(v_{j}^{C, B}\right)$ in (2.6) in terms of (twisted) Bethe equations $(2.4),(2.5)$. We denote such scalar product by $\mathcal{S}_{a, b}^{(\bar{\kappa})}$. It is easy to see that

$$
\begin{aligned}
\mathcal{S}_{a, b}^{(\bar{\kappa})}= & \sum\left(\frac{\kappa_{2}}{\kappa_{1}}\right)^{a_{\mathrm{II}}}\left(\frac{\kappa_{2}}{\kappa_{3}}\right)^{b_{\mathrm{II}}} f\left(\bar{u}_{\mathrm{II}}^{C}, \bar{u}_{\mathrm{I}}^{C}\right) f\left(\bar{u}_{\mathrm{I}}^{B}, \bar{u}_{\mathrm{II}}^{B}\right) f\left(\bar{v}_{\mathrm{I}}^{C}, \bar{v}_{\mathrm{II}}^{C}\right) f\left(\bar{v}_{\mathrm{II}}^{B}, \bar{v}_{\mathrm{I}}^{B}\right) \\
& \times f\left(\bar{v}_{\mathrm{II}}^{C}, \bar{u}_{\mathrm{II}}^{C}\right) f\left(\bar{v}_{\mathrm{I}}^{B}, \bar{u}_{\mathrm{I}}^{B}\right) Z_{a_{\mathrm{II}}, b_{\mathrm{I}}}\left(\bar{u}_{\mathrm{II}}^{C} ; \bar{u}_{\mathrm{II}}^{B} \mid \bar{v}_{\mathrm{I}}^{C} ; \bar{v}_{\mathrm{I}}^{B}\right) Z_{a_{\mathrm{I}}, b_{\mathrm{II}}}\left(\bar{u}_{\mathrm{I}}^{B} ; \bar{u}_{\mathrm{I}}^{C} \mid \bar{v}_{\mathrm{II}}^{B} ; \bar{v}_{\mathrm{II}}^{C}\right) .
\end{aligned}
$$

Remark 2.1. One should be careful using Bethe equations in the sums over partitions, because there might be problems if some parameters from the sets $\bar{u}^{C}, \bar{v}^{C}$ coincide with the ones from $\bar{u}^{B}, \bar{v}^{B}$ (i.e., $\bar{u}^{C} \cap \bar{u}^{B} \neq \varnothing$ or/and $\bar{v}^{C} \cap \bar{v}^{B} \neq \varnothing$ ). The matter is that after imposing the Bethe equations we cannot consider the limit where one solution of Bethe equations goes to another solution. Instead one should first take the limit in (2.6) (what leads to the appearance of the derivatives $r_{1}^{\prime}(u)$ and $\left.r_{3}^{\prime}(v)\right)$ and only then impose Bethe equations. However, if we consider the scalar product of twisted and usual on-shell vectors, then we can use the twisted and usual Bethe equations from the very beginning. The matter is that in this case the parameters $\bar{u}^{C}$ and $\bar{v}^{C}$ are functions of $\bar{\kappa}=\left\{\kappa_{1}, \kappa_{2}, \kappa_{3}\right\}: \bar{u}^{C}=\bar{u}^{C}(\bar{\kappa})$ and $\bar{v}^{C}=\bar{v}^{C}(\bar{\kappa})$. Therefore we always can take $\bar{\kappa}$ such that $\bar{u}^{C} \cap \bar{u}^{B}=\varnothing$ and $\bar{v}^{C} \cap \bar{v}^{B}=\varnothing$. Then, if necessary, we can consider the limit where some parameters coincide. In this case we should treat the variables $\bar{u}^{C}$ and $\bar{v}^{C}$ as functions of $\bar{\kappa}$. 
The sum over partitions (2.7) was studied in [2,4] for arbitrary values of the Bethe parameters. There this sum was reduced to a single determinant in the case $\kappa_{1}=\kappa_{3}$. It was proved that the determinant vanishes at ${ }^{4} \bar{\kappa}=1$. Thus, if $\bar{u}^{C, B} \neq \varnothing$ or $\bar{v}^{C, B} \neq \varnothing$, then setting $\bar{\kappa}=1$ in (2.7) we obtain an identity

$$
\begin{aligned}
0= & \sum f\left(\bar{u}_{\mathrm{II}}^{C}, \bar{u}_{\mathrm{I}}^{C}\right) f\left(\bar{u}_{\mathrm{I}}^{B}, \bar{u}_{\mathrm{II}}^{B}\right) f\left(\bar{v}_{\mathrm{I}}^{C}, \bar{v}_{\mathrm{II}}^{C}\right) f\left(\bar{v}_{\mathrm{II}}^{B}, \bar{v}_{\mathrm{I}}^{B}\right) f\left(\bar{v}_{\mathrm{II}}^{C}, \bar{u}_{\mathrm{II}}^{C}\right) f\left(\bar{v}_{\mathrm{I}}^{B}, \bar{u}_{\mathrm{I}}^{B}\right) \\
& \times Z_{a_{\mathrm{II}}, b_{\mathrm{I}}}\left(\bar{u}_{\mathrm{II}}^{C} ; \bar{u}_{\mathrm{II}}^{B} \mid \bar{v}_{\mathrm{I}}^{C} ; \bar{v}_{\mathrm{I}}^{B}\right) Z_{a_{\mathrm{I}}, b_{\mathrm{II}}}\left(\bar{u}_{\mathrm{I}}^{B} ; \bar{u}_{\mathrm{I}}^{C} \mid \bar{v}_{\mathrm{II}}^{B} ; \bar{v}_{\mathrm{II}}^{C}\right) .
\end{aligned}
$$

In the exceptional case $\bar{u}^{C, B}=\bar{v}^{C, B}=\varnothing$ we have

$$
\left.\mathcal{S}_{0,0}^{(\bar{\kappa})}\right|_{\bar{\kappa}=1}=1 .
$$

Remark 2.2. One should not be surprised that the r.h.s. of (2.8) does not give the norm of an on-shell Bethe vector in the case $\left\{\bar{u}^{C}, \bar{v}^{C}\right\}=\left\{\bar{u}^{B}, \bar{v}^{B}\right\}$. Indeed, as we explained above, in order to obtain the norm one should consider the limit $\left\{\bar{u}^{C}(\bar{\kappa}), \bar{v}^{C}(\bar{\kappa})\right\} \rightarrow\left\{\bar{u}^{B}, \bar{v}^{B}\right\}$ at $\bar{\kappa} \rightarrow 1$ in (2.7). Instead we simply set $\bar{\kappa}=1$ in (2.7) for generic values of the Bethe parameters. In this case we obtain that the sum (2.8) vanishes [2], and then this result can be continued to the point $\left\{\bar{u}^{C}, \bar{v}^{C}\right\}=\left\{\bar{u}^{B}, \bar{v}^{B}\right\}$.

Identity (2.8) plays a central role in the calculation of form factors of local operators.

\subsection{Universal form factors}

Form factors of the monodromy matrix entries are defined as

$$
\mathcal{F}_{a, b}^{(i, j)}(z) \equiv \mathcal{F}_{a, b}^{(i, j)}\left(z \mid \bar{u}^{C}, \bar{v}^{C} ; \bar{u}^{B}, \bar{v}^{B}\right)=\mathbb{C}^{a^{\prime}, b^{\prime}}\left(\bar{u}^{C} ; \bar{v}^{C}\right) T_{i j}(z) \mathbb{B}^{a, b}\left(\bar{u}^{B} ; \bar{v}^{B}\right),
$$

where both $\mathbb{C}^{a^{\prime}, b^{\prime}}\left(\bar{u}^{C} ; \bar{v}^{C}\right)$ and $\mathbb{B}^{a, b}\left(\bar{u}^{B} ; \bar{v}^{B}\right)$ are on-shell Bethe vectors, and

$$
a^{\prime}=a+\delta_{i 1}-\delta_{j 1}, \quad b^{\prime}=b+\delta_{j 3}-\delta_{i 3} .
$$

The parameter $z$ is an arbitrary complex number.

It was proved in [30] that if $\left\{\bar{u}^{C}, \bar{v}^{C}\right\} \neq\left\{\bar{u}^{B}, \bar{v}^{B}\right\}$, then the combination

$$
\mathfrak{F}_{a, b}^{(i, j)}\left(\bar{u}^{C}, \bar{v}^{C} ; \bar{u}^{B}, \bar{v}^{B}\right)=\frac{\mathcal{F}_{a, b}^{(i, j)}\left(z \mid \bar{u}^{C}, \bar{v}^{C} ; \bar{u}^{B}, \bar{v}^{B}\right)}{\tau\left(z \mid \bar{u}^{C}, \bar{v}^{C}\right)-\tau\left(z \mid \bar{u}^{B}, \bar{v}^{B}\right)}
$$

does not depend on $z$. We call $\mathfrak{F}_{a, b}^{(i, j)}\left(\bar{u}^{C}, \bar{v}^{C} ; \bar{u}^{B}, \bar{v}^{B}\right)$ the universal form factor of the operator $T_{i j}(z)$. If $\bar{u}^{C} \cap \bar{u}^{B}=\varnothing$ and $\bar{v}^{C} \cap \bar{v}^{B}=\varnothing$, then the universal form factor is determined by the $R$-matrix only. It does not depend on a specific model, in particular, on the functions $r_{1}(z)$ and $r_{3}(z)$.

\section{Composite generalized model}

Consider a composite generalized model defined by (1.4), (1.5). Every $T^{(l)}(u)$ satisfies $R T T$ relation (1.1) and has its own vacuum state $|0\rangle^{(l)}$. Hereby $|0\rangle=|0\rangle^{(1)} \otimes|0\rangle^{(2)}$. The operators $T_{i j}^{(2)}(u)$ and $T_{k l}^{(1)}(v)$ commute with each other, as they act in different spaces.

Let

$$
T_{i i}^{(l)}(u)|0\rangle^{(l)}=\lambda_{i}^{(l)}(u)|0\rangle^{(l)}, \quad l=1,2 .
$$

\footnotetext{
${ }^{4}$ Here and below the notation $\bar{\kappa}=1$ means $\kappa_{i}=1, i=1,2,3$.
} 
We also introduce

$$
r_{k}^{(l)}(u)=\frac{\lambda_{k}^{(l)}(u)}{\lambda_{2}^{(l)}(u)} \quad l=1,2, \quad k=1,3 .
$$

Obviously

$$
\lambda_{i}(u)=\lambda_{i}^{(1)}(u) \lambda_{i}^{(2)}(u), \quad r_{k}(u)=r_{k}^{(1)}(u) r_{k}^{(2)}(u) .
$$

Below we express form factors in terms of $r_{k}^{(1)}(u)$, therefore we introduce a special notation for these functions

$$
r_{k}^{(1)}(u)=\ell_{k}(u), \quad \text { and hence, } \quad r_{k}^{(2)}(u)=\frac{r_{k}(u)}{\ell_{k}(u)}, \quad k=1,3 .
$$

Observe that (1.6) implies

$$
\ell_{k}(u)=1+\ell_{k}[0] \frac{c}{u}+o\left(u^{-1}\right),
$$

and due to $(1.5)$

$$
r_{k}(u)=1+r_{k}[0] \frac{c}{u}+o\left(u^{-1}\right) .
$$

We extend convention (2.3) to the products of the functions $r_{k}^{(l)}(u)$ and $\ell_{k}(u)$. Namely, whenever these functions depend on sets of variables (for instance $r_{k}^{(l)}(\bar{u})$ or $\ell_{k}\left(\bar{v}_{\text {II }}\right)$ ) this means the product over the corresponding set.

\subsection{Bethe vectors and partial Bethe vectors}

We can introduce partial Bethe vectors $\mathbb{B}_{a, b}^{(l)}(\bar{u} ; \bar{v})$ for both partial monodromy matrices $T^{(l)}(u)$. Then Bethe vectors of the total monodromy matrix can be expressed in terms of the partial Bethe vectors as follows [6, 29]:

$$
\mathbb{B}_{a, b}(\bar{u} ; \bar{v})=\sum r_{1}^{(2)}\left(\bar{u}_{\mathrm{I}}\right) r_{3}^{(1)}\left(\bar{v}_{\mathrm{II}}\right) \frac{f\left(\bar{u}_{\mathrm{II}}, \bar{u}_{\mathrm{I}}\right) f\left(\bar{v}_{\mathrm{II}}, \bar{v}_{\mathrm{I}}\right)}{f\left(\bar{v}_{\mathrm{II}}, \bar{u}_{\mathrm{I}}\right)} \mathbb{B}_{a_{\mathrm{I}}, b_{\mathrm{I}}}^{(1)}\left(\bar{u}_{\mathrm{I}} ; \bar{v}_{\mathrm{I}}\right) \mathbb{B}_{a_{\mathrm{II}}, b_{\mathrm{II}}}^{(2)}\left(\bar{u}_{\mathrm{II}} ; \bar{v}_{\mathrm{II}}\right) .
$$

The sum is taken over partitions $\bar{u} \Rightarrow\left\{\bar{u}_{\mathrm{I}}, \bar{u}_{\mathrm{II}}\right\}$ and $\bar{v} \Rightarrow\left\{\bar{v}_{\mathrm{I}}, \bar{v}_{\mathrm{II}}\right\}$. The cardinalities of the subsets are given by the subscripts of the Bethe vectors.

We can present the product of functions $r_{1}^{(2)}\left(\bar{u}_{\mathrm{I}}\right)$ as $r_{1}^{(2)}\left(\bar{u}_{\mathrm{I}}\right)=r_{1}\left(\bar{u}_{\mathrm{I}}\right) \ell_{1}^{-1}\left(\bar{u}_{\mathrm{I}}\right)$, see (3.2). Moreover, if we deal with an on-shell Bethe vector, we can express $r_{1}\left(\bar{u}_{\mathrm{I}}\right)$ in terms of the function $f$, thanks to the Bethe equations (2.4). Then we obtain

$$
\mathbb{B}_{a, b}(\bar{u} ; \bar{v})=\sum \frac{\ell_{3}\left(\bar{v}_{\mathrm{II}}\right)}{\ell_{1}\left(\bar{u}_{\mathrm{I}}\right)} f\left(\bar{u}_{\mathrm{I}}, \bar{u}_{\mathrm{II}}\right) f\left(\bar{v}_{\mathrm{II}}, \bar{v}_{\mathrm{I}}\right) f\left(\bar{v}_{\mathrm{I}}, \bar{u}_{\mathrm{I}}\right) \mathbb{B}_{a_{\mathrm{I}}, b_{\mathrm{I}}}^{(1)}\left(\bar{u}_{\mathrm{I}} ; \bar{v}_{\mathrm{I}}\right) \mathbb{B}_{a_{\mathrm{II}}, b_{\mathrm{II}}}^{(2)}\left(\bar{u}_{\mathrm{II}} ; \bar{v}_{\mathrm{II}}\right) .
$$

Similarly, dual Bethe vectors can be expressed in terms of partial dual Bethe vectors

$$
\mathbb{C}_{a, b}(\bar{u} ; \bar{v})=\sum r_{1}^{(1)}\left(\bar{u}_{\mathrm{II}}\right) r_{3}^{(2)}\left(\bar{v}_{\mathrm{I}}\right) \frac{f\left(\bar{u}_{\mathrm{I}}, \bar{u}_{\mathrm{II}}\right) f\left(\bar{v}_{\mathrm{I}}, \bar{v}_{\mathrm{II}}\right)}{f\left(\bar{v}_{\mathrm{I}}, \bar{u}_{\mathrm{II}}\right)} \mathbb{C}_{a_{\mathrm{I}}, b_{\mathrm{I}}}^{(1)}\left(\bar{u}_{\mathrm{I}} ; \bar{v}_{\mathrm{I}}\right) \mathbb{C}_{a_{\mathrm{II}}, b_{\mathrm{II}}}^{(2)}\left(\bar{u}_{\mathrm{II}} ; \bar{v}_{\mathrm{II}}\right),
$$

where the sum is taken again over partitions $\bar{u} \Rightarrow\left\{\bar{u}_{\mathrm{I}}, \bar{u}_{\mathrm{II}}\right\}$ and $\bar{v} \Rightarrow\left\{\bar{v}_{\mathrm{I}}, \bar{v}_{\mathrm{II}}\right\}$.

If $\mathbb{C}_{a, b}(\bar{u} ; \bar{v})$ is a twisted on-shell Bethe vector, then we can use again $(3.2)$ as $r_{3}^{(2)}\left(\bar{v}_{\mathrm{I}}\right)=$ $r_{3}\left(\bar{v}_{\mathrm{I}}\right) \ell_{3}^{-1}\left(\bar{v}_{\mathrm{I}}\right)$ and express $r_{3}\left(\bar{v}_{\mathrm{I}}\right)$ through the twisted Bethe equations (2.5). We get

$$
\mathbb{C}_{a, b}^{(\bar{\kappa})}(\bar{u} ; \bar{v})=\sum\left(\frac{\kappa_{2}}{\kappa_{3}}\right)^{b_{\mathrm{I}}} \frac{\ell_{1}\left(\bar{u}_{\mathrm{II}}\right)}{\ell_{3}\left(\bar{v}_{\mathrm{I}}\right)} f\left(\bar{u}_{\mathrm{I}}, \bar{u}_{\mathrm{II}}\right) f\left(\bar{v}_{\mathrm{II}}, \bar{v}_{\mathrm{I}}\right) f\left(\bar{v}_{\mathrm{I}}, \bar{u}_{\mathrm{I}}\right) \mathbb{C}_{a_{\mathrm{I}}, b_{\mathrm{I}}}^{(1)}\left(\bar{u}_{\mathrm{I}} ; \bar{v}_{\mathrm{I}}\right) \mathbb{C}_{a_{\mathrm{II}}, b_{\mathrm{II}}}^{(2)}\left(\bar{u}_{\mathrm{I}} ; \bar{v}_{\mathrm{II}}\right) .
$$

Here we have added the superscript $(\bar{\kappa})$ to the vector $\mathbb{C}_{a, b}^{(\bar{\kappa})}(\bar{u} ; \bar{v})$ in order to stress that it is a twisted dual on-shell Bethe vector. 


\subsection{The action of total and partial zero modes}

The action of the operators $T_{i j}^{(l)}(z)$ on the corresponding partial Bethe vectors $\mathbb{B}_{a, b}^{(l)}(\bar{u} ; \bar{v})$ is the same as the action of total $T_{i j}(z)$ on the total Bethe vectors $\mathbb{B}_{a, b}(\bar{u} ; \bar{v})[3]$. One should only replace in the formulas the functions $r_{k}(z)$ by their partial analogs $r_{k}^{(l)}(z)$. The same replacement should be done in the action of the partial zero modes on the partial Bethe vectors [30]. In this section we give some of those actions used below.

The action of the total zero modes $T_{i j}[0]$ (with $i<j$ ) on the total Bethe vectors $\mathbb{B}_{a, b}$ can be easily extracted from the formulas given in Appendix A of the first part of this paper [29] using expansion of the monodromy matrix elements (1.6). They are

$$
\begin{aligned}
& T_{13}[0] \mathbb{B}_{a, b}(\bar{u} ; \bar{v})=\lim _{w \rightarrow \infty} \frac{w}{c} \mathbb{B}_{a+1, b+1}(\{w, \bar{u}\} ;\{w, \bar{v}\}), \\
& T_{12}[0] \mathbb{B}_{a, b}(\bar{u} ; \bar{v})=\lim _{w \rightarrow \infty} \frac{w}{c} \mathbb{B}_{a+1, b}(\{w, \bar{u}\} ; \bar{v}), \\
& T_{23}[0] \mathbb{B}_{a, b}(\bar{u} ; \bar{v})=\lim _{w \rightarrow \infty} \frac{w}{c} \mathbb{B}_{a, b+1}(\bar{u} ;\{w, \bar{v}\}) .
\end{aligned}
$$

The right action of the operators $T_{j i}[0]$ with $i<j$ on dual Bethe vectors is quite analogous. One should replace in (3.8) $T_{i j}[0]$ by $T_{j i}[0]$ and $\mathbb{B}_{a, b}(\bar{u} ; \bar{v})$ by $\mathbb{C}_{a, b}(\bar{u} ; \bar{v})$.

The action of the partial zero modes $T_{i j}^{(1)}[0]$ (with $i<j$ ) on the partial Bethe vectors $\mathbb{B}_{a, b}^{(1)}$ is similar to (3.8):

$$
\begin{aligned}
& T_{13}^{(1)}[0] \mathbb{B}_{a, b}^{(1)}(\bar{u} ; \bar{v})=\lim _{w \rightarrow \infty} \frac{w}{c} \mathbb{B}_{a+1, b+1}^{(1)}(\{w, \bar{u}\} ;\{w, \bar{v}\}), \\
& T_{12}^{(1)}[0] \mathbb{B}_{a, b}^{(1)}(\bar{u} ; \bar{v})=\lim _{w \rightarrow \infty} \frac{w}{c} \mathbb{B}_{a+1, b}^{(1)}(\{w, \bar{u}\} ; \bar{v}), \\
& T_{23}^{(1)}[0] \mathbb{B}_{a, b}^{(1)}(\bar{u} ; \bar{v})=\lim _{w \rightarrow \infty} \frac{w}{c} \mathbb{B}_{a, b+1}^{(1)}(\bar{u} ;\{w, \bar{v}\}) .
\end{aligned}
$$

The action of the partial zero modes $T_{i i}^{(1)}[0]$ has the following form

$$
\begin{aligned}
& T_{11}^{(1)}[0] \mathbb{B}_{a, b}^{(1)}(\bar{u} ; \bar{v})=\left(\ell_{1}[0]-a\right) \mathbb{B}_{a, b}^{(1)}(\bar{u} ; \bar{v}), \\
& T_{22}^{(1)}[0] \mathbb{B}_{a, b}^{(1)}(\bar{u} ; \bar{v})=(a-b) \mathbb{B}_{a, b}^{(1)}(\bar{u} ; \bar{v}), \\
& T_{33}^{(1)}[0] \mathbb{B}_{a, b}^{(1)}(\bar{u} ; \bar{v})=\left(\ell_{3}[0]+b\right) \mathbb{B}_{a, b}^{(1)}(\bar{u} ; \bar{v}),
\end{aligned}
$$

where $\ell_{k}[0]$ are determined by (3.3). In all the formulas above Bethe vectors (partial or total) are generic.

In Section 6 we will also use singular properties of on-shell (dual) Bethe vectors

$$
\mathbb{C}_{a, b}\left(\bar{u}^{C} ; \bar{v}^{C}\right) T_{i j}[0]=0, \quad T_{j i}[0] \mathbb{B}_{a, b}\left(\bar{u}^{C} ; \bar{v}^{C}\right)=0, \quad i<j .
$$

Here $\mathbb{C}_{a, b}\left(\bar{u}^{C} ; \bar{v}^{C}\right)$ and $\mathbb{B}_{a, b}\left(\bar{u}^{C} ; \bar{v}^{C}\right)$ are on-shell Bethe vectors. This property was found in [26] for $\mathrm{GL}(N)$-invariant models. In the GL(3) case it also follows from the explicit formulas of the action of the operators $T_{i j}(z)$ onto Bethe vectors [3].

\section{Main results}

Theorem 4.1. Let $\mathbb{C}_{a^{\prime}, b^{\prime}}\left(\bar{u}^{C} ; \bar{v}^{C}\right)$ and $\mathbb{B}_{a, b}\left(\bar{u}^{B} ; \bar{v}^{B}\right)$ be total on-shell vectors such that $\left\{\bar{u}^{C}, \bar{v}^{C}\right\} \neq$ $\left\{\bar{u}^{B}, \bar{v}^{B}\right\}$ (that is, these on-shell vectors have different eigenvalues). Then

$$
\mathbb{C}_{a^{\prime}, b^{\prime}}\left(\bar{u}^{C} ; \bar{v}^{C}\right) T_{i j}^{(1)}[0] \mathbb{B}_{a, b}\left(\bar{u}^{B} ; \bar{v}^{B}\right)=\left(\frac{\ell_{1}\left(\bar{u}^{C}\right) \ell_{3}\left(\bar{v}^{B}\right)}{\ell_{1}\left(\bar{u}^{B}\right) \ell_{3}\left(\bar{v}^{C}\right)}-1\right) \mathfrak{F}_{a, b}^{(i, j)}\left(\bar{u}^{C}, \bar{v}^{C} ; \bar{u}^{B}, \bar{v}^{B}\right),
$$

where $\mathfrak{F}_{a, b}^{(i, j)}$ is the universal form factor of the total operator $T_{i j}(z)$ and $a^{\prime}=a+\delta_{i 1}-\delta_{j 1}$, $b^{\prime}=b+\delta_{j 3}-\delta_{i 3}$. 
Theorem 4.2. Let $\mathbb{B}_{a, b}(\bar{u} ; \bar{v})$ be a total on-shell vector and $\mathbb{C}_{a, b}(\bar{u} ; \bar{v})$ its dual on-shell vector. Let $\mathbb{C}_{a, b}(\bar{u}(\bar{\kappa}) ; \bar{v}(\bar{\kappa}))$ be a deformation of $\mathbb{C}_{a, b}(\bar{u} ; \bar{v})$ such that the parameters $\bar{u}(\bar{\kappa})$ and $\bar{v}(\bar{\kappa})$ satisfy twisted Bethe equations (2.5), and $\bar{u}(\bar{\kappa})=\bar{u}, \bar{v}(\bar{\kappa})=\bar{v}$ at $\bar{\kappa}=1$. Then

$$
\mathbb{C}_{a, b}(\bar{u} ; \bar{v}) T_{i i}^{(1)}[0] \mathbb{B}_{a, b}(\bar{u} ; \bar{v})=\left(\delta_{i, 1} \ell_{1}[0]+\delta_{i, 3} \ell_{3}[0]+\left.\frac{d}{d \kappa_{i}} \log \frac{\ell_{1}(\bar{u}(\bar{\kappa}))}{\ell_{3}(\bar{v}(\bar{\kappa}))}\right|_{\bar{\kappa}=1}\right)\left\|\mathbb{B}_{a, b}(\bar{u} ; \bar{v})\right\|^{2}
$$

The proofs of these theorems will be given in the next sections.

If the partial monodromy matrix $T^{(1)}(u)$ has the structure (1.4), then the functions $\ell_{k}(u)$ actually depend also on the number $m$ :

$$
\ell_{k}(u)=\prod_{n=1}^{m} \ell_{k}(u \mid n), \quad k=1,3
$$

where $\ell_{k}(u \mid n)$ are the local ratios

$$
\ell_{1}(u \mid n)=\frac{\lambda_{1}(u \mid n)}{\lambda_{2}(u \mid n)}, \quad \ell_{3}(u \mid n)=\frac{\lambda_{3}(u \mid n)}{\lambda_{2}(u \mid n)} .
$$

In (4.3), we introduced the vacuum eigenvalues of local $L$-operators $L_{n}(u)$

$$
\left(L_{n}(u)\right)_{i i}|0\rangle=\lambda_{i}(u \mid n)|0\rangle, \quad i=1,2,3 .
$$

Using (1.7) and Theorems 4.1, 4.2 we can find form factors of the local operators $\left(L_{m}[0]\right)_{i j}$, $i, j=1,2,3$. Namely, one has simply to consider the difference of two $T^{(1)}(u)$ based on $m$ and $m-1$ respectively.

If $\left\{\bar{u}^{C}, \bar{v}^{C}\right\} \neq\left\{\bar{u}^{B}, \bar{v}^{B}\right\}$, then we have

$$
\begin{aligned}
\mathbb{C}_{a^{\prime}, b^{\prime}}\left(\bar{u}^{C} ; \bar{v}^{C}\right)\left(L_{m}[0]\right)_{i j} \mathbb{B}_{a, b}\left(\bar{u}^{B} ; \bar{v}^{B}\right) \\
\quad=\left(\frac{\ell_{1}\left(\bar{u}^{C} \mid m\right) \ell_{3}\left(\bar{v}^{B} \mid m\right)}{\ell_{1}\left(\bar{u}^{B} \mid m\right) \ell_{3}\left(\bar{v}^{C} \mid m\right)}-1\right)\left(\prod_{n=1}^{m-1} \frac{\ell_{1}\left(\bar{u}^{C} \mid n\right) \ell_{3}\left(\bar{v}^{B} \mid n\right)}{\ell_{1}\left(\bar{u}^{B} \mid n\right) \ell_{3}\left(\bar{v}^{C} \mid n\right)}\right) \mathfrak{F}_{a, b}^{(i, j)}\left(\bar{u}^{C}, \bar{v}^{C} ; \bar{u}^{B}, \bar{v}^{B}\right) .
\end{aligned}
$$

If $\left\{\bar{u}^{C}, \bar{v}^{C}\right\}=\left\{\bar{u}^{B}, \bar{v}^{B}\right\}=\{\bar{u}, \bar{v}\}$, then

$$
\mathbb{C}_{a, b}(\bar{u} ; \bar{v})\left(L_{m}[0]\right)_{i j} \mathbb{B}_{a, b}(\bar{u} ; \bar{v})=\left.\frac{d}{d \kappa_{i}} \log \frac{\ell_{1}(\bar{u}(\bar{\kappa}) \mid m)}{\ell_{3}(\bar{v}(\bar{\kappa}) \mid m)}\right|_{\bar{\kappa}=1}\left\|\mathbb{B}_{a, b}(\bar{u} ; \bar{v})\right\|^{2} .
$$

If we deal with a continuum model, then form factors of local operators can be found directly from (4.1), (4.2). In this case the integer number $m$ turns into a continuous variable $x$. This parameter enters only the functions $\ell_{k}$, and taking the $x$-derivative of (4.1), (4.2) we find form factors of local operators in the point $x$.

Thus, we obtain form factors of local operators in the generalized model without use of a specific representation of the algebra (1.1). In fact, it means that we have a solution of the quantum inverse scattering problem in the weak sense. We cannot express the local operators in terms of the monodromy matrix entries as it was done in [20, 25], but we can find all their matrix elements in the basis of the transfer matrix eigenstates. Furthermore, we have determinant formulas for all these matrix elements [2, 4, 27, 28, 30].

GL $(\boldsymbol{N})$ generalisation. We would like to mention that Theorems 4.1 and 4.2 admit a direct generalization to $\mathrm{GL}(N)$-invariant models with $N>3$. Indeed, Bethe vectors (and dual ones) of $\operatorname{GL}(N)$ models depend on $N-1$ sets of parameters $\bar{t}^{j}=\left\{t_{1}^{j}, t_{2}^{j}, \ldots, t_{a_{j}}^{j}\right\}, j=1,2, \ldots, N-1$ and $N-1$ integers $a_{j}$ that correspond to the cardinalities of each set:

$$
\mathbb{B}_{\bar{a}}(\bar{t})=\mathbb{B}_{a_{1}, a_{2}, \ldots, a_{N-1}}\left(\bar{t}^{1}, \bar{t}^{2}, \ldots, \bar{t}^{N-1}\right), \quad \mathbb{C}_{\bar{a}}(\bar{t})=\mathbb{C}_{a_{1}, a_{2}, \ldots, a_{N-1}}\left(\bar{t}^{1}, \bar{t}^{2}, \ldots, \bar{t}^{N-1}\right) .
$$


The action of the diagonal entries $T_{i i}^{(l)}$ on the vacuum vectors is similar to (3.1)

$$
T_{i i}^{(l)}(t)|0\rangle^{(l)}=\lambda_{i}^{(l)}(t)|0\rangle^{(l)}, \quad l=1,2, \quad i=1, \ldots, N,
$$

and we can introduce

$$
\alpha_{i}(t)=\frac{\lambda_{i}^{(1)}(t)}{\lambda_{i+1}^{(1)}(t)}, \quad i=1, \ldots, N-1 .
$$

Note that in the case $N=3$ we have $\alpha_{1}(t)=\ell_{1}(t)$, while $\alpha_{2}(t)=\ell_{3}^{-1}(t)$.

Conjecture 4.3. Form factors of the partial zero modes $T_{i j}^{(1)}[0]$ in $\mathrm{GL}(N)$-invariant models are given by

$$
\begin{aligned}
& \mathbb{C}_{\bar{b}}(\bar{s}) T_{i j}^{(1)}[0] \mathbb{B}_{\bar{a}}(\bar{t})=\left(\prod_{k=1}^{N-1} \frac{\alpha_{k}\left(\bar{s}^{k}\right)}{\alpha_{k}\left(\bar{t}^{k}\right)}-1\right) \mathfrak{F}_{\bar{a}}^{(i, j)}(\bar{s} ; \bar{t}), \quad \text { for } \quad \bar{s} \neq \bar{t} \\
& \mathbb{C}_{\bar{a}}(\bar{t}) T_{i i}^{(1)}[0] \mathbb{B}_{\bar{a}}(\bar{t})=\left(\lambda_{i}^{(1)}[0]+\left.\sum_{k=1}^{N-1} \frac{d}{d \kappa_{i}} \log \alpha_{k}\left(\bar{t}^{k}(\bar{\kappa})\right)\right|_{\bar{\kappa}=1}\right)\left\|\mathbb{B}_{\bar{a}}(\bar{t})\right\|^{2} .
\end{aligned}
$$

where $\mathfrak{F}_{\bar{a}}^{(i, j)}$ is the universal ( $z$-independent) form factor of the total operator $T_{i j}(z)$ and we extended the convention (2.3) to the functions $\alpha_{k} . \bar{t}(\bar{\kappa})$ is a set of $\kappa$-twisted on-shell Bethe parameters, coinciding with $\bar{t}$ when $\bar{\kappa}=1$.

This conjecture generalizes Theorems 4.1 and 4.2 , proved for $N=2$ and $N=3$.

Remark that since $\mathbb{C}_{\bar{b}}(\bar{s}) T_{i j}[0] \mathbb{B}_{\bar{a}}(\bar{t})=0$ when $\bar{s} \neq \bar{t}$, the above conjecture and theorems provide also the form factors for $T_{i j}^{(2)}[0]$.

It is worth mentioning that in the cases $N=2$ and $N=3$, compact determinant representations for the universal form factors are known. In contrast, in the case $N>3$ such representations are missing up to now. Nevertheless, if Conjecture 4.3 is valid in the $\mathrm{GL}(N)$ case, then it gives explicit dependence on the lattice site $m$ of the partial zero modes form factors.

\section{Form factors of diagonal operators}

We begin our consideration with the form factors of the diagonal partial zero modes $T_{i i}^{(1)}[0]$. It is convenient to construct a special generating functional for these form factors [10]. Consider an operator

$$
Q_{\bar{\beta}}=\sum_{i=1}^{3} \beta_{i} T_{i i}^{(1)}[0],
$$

where $\beta_{i}$ are some complex numbers. The generating functional is

$$
M_{a, b}^{(\bar{\kappa})}=\mathbb{C}_{a, b}^{(\bar{\kappa})}\left(\bar{u}^{C} ; \bar{v}^{C}\right) e^{Q_{\bar{\beta}}} \mathbb{B}_{a, b}\left(\bar{u}^{B} ; \bar{v}^{B}\right) .
$$

Here $\mathbb{B}_{a, b}\left(\bar{u}^{B} ; \bar{v}^{B}\right)$ is an on-shell Bethe vector, $\mathbb{C}_{a, b}^{(\bar{\kappa})}\left(\bar{u}^{C} ; \bar{v}^{C}\right)$ is a dual twisted on-shell Bethe vector with the twist parameters $\kappa_{i}=e^{\beta_{i}}$.

Lemma 5.1. Let $M_{a, b}^{(\bar{\kappa})}$ is defined as in (5.1). Then

$$
M_{a, b}^{(\bar{\kappa})}=e^{\beta_{1} \ell_{1}[0]+\beta_{3} \ell_{3}[0]} \frac{\ell_{1}\left(\bar{u}^{C}\right) \ell_{3}\left(\bar{v}^{B}\right)}{\ell_{1}\left(\bar{u}^{B}\right) \ell_{3}\left(\bar{v}^{C}\right)} \mathcal{S}_{a, b}^{(\bar{\kappa})},
$$

where $\mathcal{S}_{a, b}^{(\bar{\kappa})}$ is the scalar product of the twisted and the usual on-shell Bethe vectors (2.7). 
It is worth mentioning that an analog of (5.2) for GL(2)-based models was obtained in [14]. We will give a proof of Lemma 5.1 in Section 5.1. Now we show how equation (5.2) implies some statements of Theorems 4.1 and 4.2 .

Differentiating (5.1) over $\kappa_{i}$ at $\bar{\kappa}=1$ we obtain

$$
\left.\frac{d}{d \kappa_{i}} M_{a, b}^{(\bar{\kappa})}\right|_{\bar{\kappa}=1}=\left.\frac{d}{d \kappa_{i}} \mathcal{S}_{a, b}^{(\bar{\kappa})}\right|_{\bar{\kappa}=1}+\left.\mathbb{C}_{a, b}^{(\bar{\kappa})}\left(\bar{u}^{C} ; \bar{v}^{C}\right)\right|_{\bar{\kappa}=1} T_{i i}^{(1)}[0] \mathbb{B}_{a, b}\left(\bar{u}^{B} ; \bar{v}^{B}\right)
$$

Pay attention that the dual vector $\mathbb{C}_{a, b}\left(\bar{u}^{C} ; \bar{v}^{C}\right)=\left.\mathbb{C}_{a, b}^{(\bar{\kappa})}\left(\bar{u}^{C} ; \bar{v}^{C}\right)\right|_{\bar{\kappa}=1}$ is an on-shell vector. Thus, the second term in the r.h.s. of (5.3) is a form factor of the partial zero mode $T_{i i}^{(1)}[0]$.

On the other hand, differentiating the r.h.s. of (5.2) over $\kappa_{i}$ at $\bar{\kappa}=1$ we find

$$
\begin{gathered}
\left.\frac{d}{d \kappa_{i}} e^{\beta_{1} \ell_{1}[0]+\beta_{3} \ell_{3}[0]} \frac{\ell_{1}\left(\bar{u}^{C}\right) \ell_{3}\left(\bar{v}^{B}\right)}{\ell_{1}\left(\bar{u}^{B}\right) \ell_{3}\left(\bar{v}^{C}\right)} \mathcal{S}_{a, b}^{(\bar{\kappa})}\right|_{\bar{\kappa}=1}=\left.\left.\frac{\ell_{1}\left(\bar{u}^{C}\right) \ell_{3}\left(\bar{v}^{B}\right)}{\ell_{1}\left(\bar{u}^{B}\right) \ell_{3}\left(\bar{v}^{C}\right)}\right|_{\bar{\kappa}=1} \frac{d}{d \kappa_{i}} \mathcal{S}_{a, b}^{(\bar{\kappa})}\right|_{\bar{\kappa}=1} \\
+\delta_{\mathbb{C}, \mathbb{B}}\left(\delta_{i, 1} \ell_{1}[0]+\delta_{i, 3} \ell_{3}[0]+\left.\frac{d}{d \kappa_{i}} \log \frac{\ell_{1}(\bar{u})}{\ell_{3}(\bar{v})}\right|_{\bar{\kappa}=1}\right)\left\|\mathbb{B}_{a, b}(\bar{u} ; \bar{v})\right\|^{2}
\end{gathered}
$$

where

$$
\delta_{\mathbb{C}, \mathbb{B}}= \begin{cases}1, & \text { if }\left.\left\{\bar{u}^{C}, \bar{v}^{C}\right\}\right|_{\bar{\kappa}=1}=\left\{\bar{u}^{B}, \bar{v}^{B}\right\} \\ 0, & \text { if }\left.\left\{\bar{u}^{C}, \bar{v}^{C}\right\}\right|_{\bar{\kappa}=1} \neq\left\{\bar{u}^{B}, \bar{v}^{B}\right\} .\end{cases}
$$

Deriving this formula we used the orthogonality of on-shell Bethe vectors depending on different Bethe parameters. Comparing equations (5.3) and (5.4) we immediately arrive at the statement of Theorem 4.2. If $\left.\left\{\bar{u}^{C}, \bar{v}^{C}\right\}\right|_{\bar{\kappa}=1} \neq\left\{\bar{u}^{B}, \bar{v}^{B}\right\}$, then we obtain

$$
\mathbb{C}_{a, b}\left(\bar{u}^{C} ; \bar{v}^{C}\right) T_{i i}^{(1)}[0] \mathbb{B}_{a, b}\left(\bar{u}^{B} ; \bar{v}^{B}\right)=\left.\left.\left(\frac{\ell_{1}\left(\bar{u}^{C}\right) \ell_{3}\left(\bar{v}^{B}\right)}{\ell_{1}\left(\bar{u}^{B}\right) \ell_{3}\left(\bar{v}^{C}\right)}-1\right)\right|_{\bar{\kappa}=1} \frac{d}{d \kappa_{i}} \mathcal{S}_{a, b}^{(\bar{\kappa})}\right|_{\bar{\kappa}=1} .
$$

It was proved in [4] that the $\kappa_{i}$-derivative of the scalar product $\mathcal{S}_{a, b}^{(\bar{\kappa})}$ at $\bar{\kappa}=1$ is equal to the universal form factor of the operator $T_{i i}(z)$. Thus, the statement of Theorem 4.1 is proved for partial zero modes $T_{i i}^{(1)}[0]$.

\subsection{Proof of Lemma 5.1}

The proof of Lemma 5.1 is lengthy but straightforward. Let us first sketch the general strategy before going into details. Knowing the action of the operator $e^{Q_{\bar{\beta}}}$ on the partial Bethe vectors we find its action on the total Bethe vectors. Then we can can calculate the matrix element $M_{a, b}^{(\bar{\kappa})}$ in terms of scalar products of partial Bethe vectors, for which we use equation (2.6). The resulting formula becomes rather cumbersome. In particular, it contains a sum over partitions of every set of the original Bethe parameters into four subsets. Therefore in this section we use standard arabic indices in order to label these subsets. New subsets of the Bethe parameters can be easily recombined into new sets of variables, and after this the proof reduces to the use of identity (2.8) and equation (2.7).

Let us now give the details. Using (3.10) and (3.5) we find the action of $e^{Q_{\bar{\beta}}}$ on the total on-shell Bethe vector

$$
\begin{aligned}
e^{Q_{\bar{\beta}}} \mathbb{B}_{a, b}\left(\bar{u}^{B} ; \bar{v}^{B}\right)= & \sum e^{\beta_{1}\left(\ell_{1}[0]-a_{\mathrm{I}}\right)+\beta_{2}\left(a_{\mathrm{I}}-b_{\mathrm{I}}\right)+\beta_{3}\left(\ell_{3}[0]+b_{\mathrm{I}}\right)} \frac{\ell_{3}\left(\bar{v}_{\mathrm{II}}^{B}\right)}{\ell_{1}\left(\bar{u}_{\mathrm{I}}^{B}\right)} \\
& \times f\left(\bar{u}_{\mathrm{I}}^{B}, \bar{u}_{\mathrm{II}}^{B}\right) f\left(\bar{v}_{\mathrm{II}}^{B}, \bar{v}_{\mathrm{I}}^{B}\right) f\left(\bar{v}_{\mathrm{I}}^{B}, \bar{u}_{\mathrm{I}}^{B}\right) \mathbb{B}_{a_{\mathrm{I}}, b_{\mathrm{I}}}^{(1)}\left(\bar{u}_{\mathrm{I}}^{B} ; \bar{v}_{\mathrm{I}}^{B}\right) \mathbb{B}_{a_{\mathrm{II}}, b_{\mathrm{II}}}^{(2)}\left(\bar{u}_{\mathrm{II}}^{B} ; \bar{v}_{\mathrm{II}}^{B}\right) .
\end{aligned}
$$


Multiplying this equation from the left by $\mathbb{C}_{a, b}^{(\bar{\kappa})}\left(\bar{u}^{C} ; \bar{v}^{C}\right)$ and using (3.7) we obtain

$$
\begin{aligned}
M_{a, b}^{(\bar{\kappa})}= & \sum e^{\beta_{1}\left(\ell_{1}[0]-a_{\mathrm{I}}\right)+\beta_{2} a_{\mathrm{I}}+\beta_{3} \ell_{3}[0]} \frac{\ell_{1}\left(\bar{u}_{\mathrm{I}}^{C}\right) \ell_{3}\left(\bar{v}_{\mathrm{II}}^{B}\right)}{\ell_{1}\left(\bar{u}_{\mathrm{I}}^{B}\right) \ell_{3}\left(\bar{v}_{\mathrm{I}}^{C}\right)} \\
& \times f\left(\bar{u}_{\mathrm{I}}^{C}, \bar{u}_{\mathrm{II}}^{C}\right) f\left(\bar{u}_{\mathrm{I}}^{B}, \bar{u}_{\mathrm{II}}^{B}\right) f\left(\bar{v}_{\mathrm{II}}^{C}, \bar{v}_{\mathrm{I}}^{C}\right) f\left(\bar{v}_{\mathrm{II}}^{B}, \bar{v}_{\mathrm{I}}^{B}\right) f\left(\bar{v}_{\mathrm{I}}^{B}, \bar{u}_{\mathrm{I}}^{B}\right) f\left(\bar{v}_{\mathrm{I}}^{C}, \bar{u}_{\mathrm{I}}^{C}\right) \\
& \times \mathbb{C}_{a_{\mathrm{I}}, b_{\mathrm{I}}}^{(1)}\left(\bar{u}_{\mathrm{I}}^{C} ; \bar{v}_{\mathrm{I}}^{C}\right) \mathbb{B}_{a_{\mathrm{I}}, b_{\mathrm{I}}}^{(1)}\left(\bar{u}_{\mathrm{I}}^{B} ; \bar{v}_{\mathrm{I}}^{B}\right) \cdot \mathbb{C}_{a_{\mathrm{II}}, b_{\mathrm{II}}}^{(2)}\left(\bar{u}_{\mathrm{II}}^{C} ; \bar{v}_{\mathrm{II}}^{C}\right) \mathbb{B}_{a_{\mathrm{II}}, b_{\mathrm{II}}}^{(2)}\left(\bar{u}_{\mathrm{II}}^{B} ; \bar{v}_{\mathrm{II}}^{B}\right) .
\end{aligned}
$$

Thus, we have obtained the expression for $M_{a, b}^{(\bar{\kappa})}$ in terms of scalar products of partial Bethe vectors. Note that in spite of the sets $\bar{u}^{B}$ and $\bar{v}^{B}$ satisfy the Bethe equations (2.4), and the sets $\bar{u}^{C}$ and $\bar{v}^{C}$ satisfy the twisted Bethe equations (2.5), the partial Bethe vectors in (5.5) are not (twisted) on-shell vectors. In other words we deal with the scalar products of generic Bethe vectors in (5.5). Therefore, we do not write the additional superscript $(\bar{\kappa})$ for the dual vectors and we should use (2.6) for the calculation of their scalar products. Hereby, for the scalar product of the vectors $\mathbb{C}^{(1)}$ and $\mathbb{B}^{(1)}$ we should replace in (2.6) the functions $r_{k}$ by $\ell_{k}$, while for the scalar product of the vectors $\mathbb{C}^{(2)}$ and $\mathbb{B}^{(2)}$ we should replace in (2.6) the functions $r_{k}$ by $r_{k} \ell_{k}^{-1}$.

The use of (2.6) introduces new partitions of the subsets of Bethe parameters, so that, as mentioned above (see Section 2.2), we use now arabic numbers to label the numerous subsubsets. Thus, we have for the first scalar product

$$
\begin{aligned}
& \mathbb{C}_{a, b}^{(1)}\left(\bar{u}_{\mathrm{I}}^{C} ; \bar{v}_{\mathrm{I}}^{C}\right) \mathbb{B}_{a, b}^{(1)}\left(\bar{u}_{\mathrm{I}}^{B} ; \bar{v}_{\mathrm{I}}^{B}\right)=\sum \ell_{1}\left(\bar{u}_{1}^{B}\right) \ell_{1}\left(\bar{u}_{3}^{C}\right) \ell_{3}\left(\bar{v}_{3}^{C}\right) \ell_{3}\left(\bar{v}_{1}^{B}\right) f\left(\bar{u}_{1}^{C}, \bar{u}_{3}^{C}\right) f\left(\bar{u}_{3}^{B}, \bar{u}_{1}^{B}\right) \\
& \quad \times f\left(\bar{v}_{3}^{C}, \bar{v}_{1}^{C}\right) f\left(\bar{v}_{1}^{B}, \bar{v}_{3}^{B}\right) \frac{f\left(\bar{v}_{1}^{C}, \bar{u}_{1}^{C}\right) f\left(\bar{v}_{3}^{B}, \bar{u}_{3}^{B}\right)}{f\left(\bar{v}_{\mathrm{I}}^{C}, \bar{u}_{\mathrm{I}}^{C}\right) f\left(\bar{v}_{\mathrm{I}}^{B}, \bar{u}_{\mathrm{I}}^{B}\right)} Z_{a_{3}, b_{1}}\left(\bar{u}_{3}^{C} ; \bar{u}_{3}^{B} \mid \bar{v}_{1}^{C} ; \bar{v}_{1}^{B}\right) Z_{a_{1}, b_{3}}\left(\bar{u}_{1}^{B} ; \bar{u}_{1}^{C} \mid \bar{v}_{3}^{B} ; \bar{v}_{3}^{C}\right) .
\end{aligned}
$$

The summation is taken with respect to the partitions

$$
\bar{u}_{\mathrm{I}}^{C, B} \Rightarrow\left\{\bar{u}_{1}^{C, B}, \bar{u}_{3}^{C, B}\right\}, \quad \bar{v}_{\mathrm{I}}^{C, B} \Rightarrow\left\{\bar{v}_{1}^{C, B}, \bar{v}_{3}^{C, B}\right\} .
$$

The cardinalities of the subsubsets are $a_{n}=\# \bar{u}_{n}^{C, B}, b_{n}=\# \bar{v}_{n}^{C, B}, n=1,3$.

Similarly

$$
\begin{aligned}
& \mathbb{C}_{a, b}^{(2)}\left(\bar{u}_{\mathrm{II}}^{C} ; \bar{v}_{\mathrm{II}}^{C}\right) \mathbb{B}_{a, b}^{(2)}\left(\bar{u}_{\mathrm{II}}^{B} ; \bar{v}_{\mathrm{II}}^{B}\right)=\sum \frac{r_{1}\left(\bar{u}_{2}^{B}\right) r_{1}\left(\bar{u}_{4}^{C}\right) r_{3}\left(\bar{v}_{4}^{C}\right) r_{3}\left(\bar{v}_{2}^{B}\right)}{\ell_{1}\left(\bar{u}_{2}^{B}\right) \ell_{1}\left(\bar{u}_{4}^{C}\right) \ell_{3}\left(\bar{v}_{4}^{C}\right) \ell_{3}\left(\bar{v}_{2}^{B}\right)} f\left(\bar{u}_{2}^{C}, \bar{u}_{4}^{C}\right) f\left(\bar{u}_{4}^{B}, \bar{u}_{2}^{B}\right) \\
& \quad \times f\left(\bar{v}_{4}^{C}, \bar{v}_{2}^{C}\right) f\left(\bar{v}_{2}^{B}, \bar{v}_{4}^{B}\right) \frac{f\left(\bar{v}_{2}^{C}, \bar{u}_{2}^{C}\right) f\left(\bar{v}_{4}^{B}, \bar{u}_{4}^{B}\right)}{f\left(\bar{v}_{\mathrm{II}}^{C}, \bar{u}_{\mathrm{II}}^{C}\right) f\left(\bar{v}_{\mathrm{II}}^{B}, \bar{u}_{\mathrm{II}}^{B}\right)} Z_{a_{4}, b_{2}}\left(\bar{u}_{4}^{C} ; \bar{u}_{4}^{B} \mid \bar{v}_{2}^{C} ; \bar{v}_{2}^{B}\right) Z_{a_{2}, b_{4}}\left(\bar{u}_{2}^{B} ; \bar{u}_{2}^{C} \mid \bar{v}_{4}^{B} ; \bar{v}_{4}^{C}\right) .
\end{aligned}
$$

Here the sum is taken over partitions

$$
\bar{u}_{\mathrm{II}}^{C, B} \Rightarrow\left\{\bar{u}_{2}^{C, B}, \bar{u}_{4}^{C, B}\right\}, \quad \bar{v}_{\mathrm{II}}^{C, B} \Rightarrow\left\{\bar{v}_{2}^{C, B}, \bar{v}_{4}^{C, B}\right\} .
$$

The cardinalities of the subsubsets are still denoted by $a_{n}=\# \bar{u}_{n}^{C, B}$, and $b_{n}=\# \bar{v}_{n}^{C, B}, n=2,4$.

Now we should express the products of the functions $r_{k}$ in (5.6) via the (twisted) Bethe equations for the full sets $\left\{\bar{u}^{B}, \bar{v}^{B}\right\}$ and $\left\{\bar{u}^{C}, \bar{v}^{C}\right\}$. We have

$$
\begin{aligned}
r_{1}\left(\bar{u}_{2}^{B}\right) & =\frac{f\left(\bar{u}_{2}^{B}, \bar{u}_{1}^{B}\right) f\left(\bar{u}_{2}^{B}, \bar{u}_{3}^{B}\right) f\left(\bar{u}_{2}^{B}, \bar{u}_{4}^{B}\right)}{f\left(\bar{u}_{1}^{B}, \bar{u}_{2}^{B}\right) f\left(\bar{u}_{3}^{B}, \bar{u}_{2}^{B}\right) f\left(\bar{u}_{4}^{B}, \bar{u}_{2}^{B}\right)} f\left(\bar{v}^{B}, \bar{u}_{2}^{B}\right), \\
r_{3}\left(\bar{v}_{2}^{B}\right) & =\frac{f\left(\bar{v}_{1}^{B}, \bar{v}_{2}^{B}\right) f\left(\bar{v}_{3}^{B}, \bar{v}_{2}^{B}\right) f\left(\bar{v}_{4}^{B}, \bar{v}_{2}^{B}\right)}{f\left(\bar{v}_{2}^{B}, \bar{v}_{1}^{B}\right) f\left(\bar{v}_{2}^{B}, \bar{v}_{3}^{B}\right) f\left(\bar{v}_{2}^{B}, \bar{v}_{4}^{B}\right)} f\left(\bar{v}_{2}^{B}, \bar{u}^{B}\right), \\
r_{1}\left(\bar{u}_{4}^{C}\right) & =e^{a_{4}\left(\beta_{2}-\beta_{1}\right)} \frac{f\left(\bar{u}_{4}^{C}, \bar{u}_{1}^{C}\right) f\left(\bar{u}_{4}^{C}, \bar{u}_{2}^{C}\right) f\left(\bar{u}_{4}^{C}, \bar{u}_{3}^{C}\right)}{f\left(\bar{u}_{1}^{C}, \bar{u}_{4}^{C}\right) f\left(\bar{u}_{2}^{C}, \bar{u}_{4}^{C}\right) f\left(\bar{u}_{3}^{C}, \bar{u}_{4}^{C}\right)} f\left(\bar{v}^{C}, \bar{u}_{4}^{C}\right), \\
r_{3}\left(\bar{v}_{4}^{C}\right) & =e^{b_{4}\left(\beta_{2}-\beta_{3}\right)} \frac{f\left(\bar{v}_{1}^{C}, \bar{v}_{4}^{C}\right) f\left(\bar{v}_{2}^{C}, \bar{v}_{4}^{C}\right) f\left(\bar{v}_{3}^{C}, \bar{v}_{4}^{C}\right)}{f\left(\bar{v}_{4}^{C}, \bar{v}_{1}^{C}\right) f\left(\bar{v}_{4}^{C}, \bar{v}_{2}^{C}\right) f\left(\bar{v}_{4}^{C}, \bar{v}_{3}^{C}\right)} f\left(\bar{v}_{4}^{C}, \bar{u}^{C}\right) .
\end{aligned}
$$


All these expressions should be substituted into (5.5). After simple but exhausting algebra we obtain

$$
\begin{aligned}
M_{a, b}^{(\bar{\kappa})}= & \sum e^{\beta_{1} \ell_{1}[0]+\beta_{3} \ell_{3}[0]+\left(\beta_{2}-\beta_{1}\right)\left(a-a_{2}\right)+\left(\beta_{2}-\beta_{3}\right) b_{4}} \frac{\ell_{1}\left(\bar{u}_{2}^{C}\right) \ell_{1}\left(\bar{u}_{3}^{C}\right) \ell_{3}\left(\bar{v}_{1}^{B}\right) \ell_{3}\left(\bar{v}_{4}^{B}\right)}{\ell_{1}\left(\bar{u}_{2}^{B}\right) \ell_{1}\left(\bar{u}_{3}^{B}\right) \ell_{3}\left(\bar{v}_{1}^{C}\right) \ell_{3}\left(\bar{v}_{4}^{C}\right)} \\
& \times F_{u u}^{C} F_{v v}^{C} F_{v u}^{C} F_{u u}^{B} F_{v v}^{B} F_{v u}^{B} \mathcal{Z} .
\end{aligned}
$$

Here the sum is taken over partitions of every set of Bethe parameters into four subsets

$$
\bar{u}^{C, B} \Rightarrow\left\{\bar{u}_{1}^{C, B}, \bar{u}_{2}^{C, B}, \bar{u}_{3}^{C, B}, \bar{u}_{4}^{C, B}\right\}, \quad \bar{v}^{C, B} \Rightarrow\left\{\bar{v}_{1}^{C, B}, \bar{v}_{2}^{C, B}, \bar{v}_{3}^{C, B}, \bar{v}_{4}^{C, B}\right\} .
$$

We have $\# \bar{u}_{n}^{B}=\# \bar{u}_{n}^{C}=a_{n}$ and $\# \bar{v}_{n}^{B}=\# \bar{v}_{n}^{C}=b_{n}, n=1, \ldots, 4$, but the values $a_{n}$ and $b_{n}$ are free. Note that $a_{2}$ and $b_{4}$ explicitly appear as coefficients in (5.7), so that manipulations with $\bar{u}_{2}^{C, B}$ and $\bar{v}_{4}^{C, B}$ should include these coefficients.

The factor $\mathcal{Z}$ in (5.7) is the product of four highest coefficients

$$
\mathcal{Z}=Z_{a_{3}, b_{1}}\left(\bar{u}_{3}^{C} ; \bar{u}_{3}^{B} \mid \bar{v}_{1}^{C} ; \bar{v}_{1}^{B}\right) Z_{a_{1}, b_{3}}\left(\bar{u}_{1}^{B} ; \bar{u}_{1}^{C} \mid \bar{v}_{3}^{B} ; \bar{v}_{3}^{C}\right) Z_{a_{4}, b_{2}}\left(\bar{u}_{4}^{C} ; \bar{u}_{4}^{B} \mid \bar{v}_{2}^{C} ; \bar{v}_{2}^{B}\right) Z_{a_{2}, b_{4}}\left(\bar{u}_{2}^{B} ; \bar{u}_{2}^{C} \mid \bar{v}_{4}^{B} ; \bar{v}_{4}^{C}\right) .
$$

The other factors in (5.7) denoted by $F$ with different subscripts and superscripts are products of $f$ functions:

$$
\begin{aligned}
& F_{u u}^{C}=f\left(\bar{u}_{4}^{C}, \bar{u}_{1}^{C}\right) f\left(\bar{u}_{3}^{C}, \bar{u}_{2}^{C}\right) f\left(\bar{u}_{4}^{C}, \bar{u}_{2}^{C}\right) f\left(\bar{u}_{4}^{C}, \bar{u}_{3}^{C}\right) f\left(\bar{u}_{1}^{C}, \bar{u}_{2}^{C}\right) f\left(\bar{u}_{1}^{C}, \bar{u}_{3}^{C}\right), \\
& F_{u u}^{B}=f\left(\bar{u}_{1}^{B}, \bar{u}_{4}^{B}\right) f\left(\bar{u}_{2}^{B}, \bar{u}_{3}^{B}\right) f\left(\bar{u}_{2}^{B}, \bar{u}_{1}^{B}\right) f\left(\bar{u}_{2}^{B}, \bar{u}_{1}^{B}\right) f\left(\bar{u}_{3}^{B}, \bar{u}_{4}^{B}\right) f\left(\bar{u}_{3}^{B}, \bar{u}_{4}^{B}\right), \\
& F_{v v}^{C}=f\left(\bar{v}_{1}^{C}, \bar{v}_{4}^{C}\right) f\left(\bar{v}_{2}^{C}, \bar{v}_{3}^{C}\right) f\left(\bar{v}_{2}^{C}, \bar{v}_{1}^{C}\right) f\left(\bar{v}_{2}^{C}, \bar{v}_{4}^{C}\right) f\left(\bar{v}_{3}^{C}, \bar{v}_{1}^{C}\right) f\left(\bar{v}_{3}^{C}, \bar{v}_{4}^{C}\right), \\
& F_{v v}^{B}=f\left(\bar{v}_{4}^{B}, \bar{v}_{1}^{B}\right) f\left(\bar{v}_{3}^{B}, \bar{v}_{2}^{B}\right) f\left(\bar{v}_{1}^{B}, \bar{v}_{3}^{B}\right) f\left(\bar{v}_{4}^{B}, \bar{v}_{3}^{B}\right) f\left(\bar{v}_{1}^{B}, \bar{v}_{2}^{B}\right) f\left(\bar{v}_{4}^{B}, \bar{v}_{2}^{B}\right), \\
& F_{v u}^{C}=f\left(\bar{v}_{1}^{C}, \bar{u}_{4}^{C}\right) f\left(\bar{v}_{4}^{C}, \bar{u}_{4}^{C}\right) f\left(\bar{v}_{1}^{C}, \bar{u}_{1}^{C}\right) f\left(\bar{v}_{4}^{C}, \bar{u}_{1}^{C}\right) f\left(\bar{v}_{3}^{C}, \bar{u}_{4}^{C}\right) f\left(\bar{v}_{4}^{C}, \bar{u}_{3}^{C}\right), \\
& F_{v u}^{B}=f\left(\bar{v}_{3}^{B}, \bar{u}_{3}^{B}\right) f\left(\bar{v}_{2}^{B}, \bar{u}_{2}^{B}\right) f\left(\bar{v}_{3}^{B}, \bar{u}_{2}^{B}\right) f\left(\bar{v}_{2}^{B}, \bar{u}_{3}^{B}\right) f\left(\bar{v}_{1}^{B}, \bar{u}_{2}^{B}\right) f\left(\bar{v}_{2}^{B}, \bar{u}_{1}^{B}\right) .
\end{aligned}
$$

It remains to combine the subsubsets into new groups:

$$
\begin{array}{ll}
\left\{\bar{u}_{1}^{C, B}, \bar{u}_{4}^{C, B}\right\}=\bar{u}_{\mathrm{i}}^{C, B}, & \left\{\bar{u}_{2}^{C, B}, \bar{u}_{3}^{C, B}\right\}=\bar{u}_{\mathrm{ii}}^{C, B}, \\
\left\{\bar{v}_{1}^{C, B}, \bar{v}_{4}^{C, B}\right\}=\bar{v}_{\mathrm{i}}^{C, B}, & \left\{\bar{v}_{2}^{C, B}, \bar{v}_{3}^{C, B}\right\}=\bar{v}_{\mathrm{ii}}^{C, B} .
\end{array}
$$

Then we recast (5.7) as follows:

$$
\begin{aligned}
M_{a, b}^{(\bar{\kappa})}= & \sum_{\substack{\bar{u}^{C, B} \Rightarrow\left\{\bar{u}_{\mathrm{i}}^{C, B}, \bar{u}_{\mathrm{ii}}^{C}, B\\
\\
\right.}} \frac{\ell_{1}\left(\bar{u}_{\mathrm{ii}}^{C}\right) \ell_{3}\left(\bar{v}_{\mathrm{i}}^{B}\right)}{\ell_{1}\left(\bar{u}_{\mathrm{ii}}^{B}\right) \ell_{3}\left(\bar{v}_{\mathrm{i}}^{C}\right)} f\left(\bar{u}_{\mathrm{i}}^{C}, \bar{u}_{\mathrm{ii}}^{C}\right) f\left(\bar{u}_{\mathrm{ii}}^{B}, \bar{u}_{\mathrm{i}}^{B}\right) f\left(\bar{v}_{\mathrm{ii}}^{C}, \bar{v}_{\mathrm{i}}^{C}\right) f\left(\bar{v}_{\mathrm{i}}^{B}, \bar{v}_{\mathrm{ii}}^{B}\right) \\
& \times f\left(\bar{v}_{\mathrm{i}}^{C}, \bar{u}_{\mathrm{i}}^{C}\right) f\left(\bar{v}_{\mathrm{ii}}^{B}, \bar{u}_{\mathrm{ii}}^{B}\right) G_{1}\left(\bar{u}_{\mathrm{i}}^{C}, \bar{u}_{\mathrm{i}}^{B} ; \bar{v}_{\mathrm{ii}}^{C}, \bar{v}_{\mathrm{ii}}^{B}\right) G_{2}\left(\bar{u}_{\mathrm{ii}}^{C}, \bar{u}_{\mathrm{ii}}^{B} ; \bar{v}_{\mathrm{i}}^{C}, \bar{v}_{\mathrm{i}}^{B}\right),
\end{aligned}
$$

where factors $G_{1}$ and $G_{2}$ are given as sums over partitions

$$
\begin{aligned}
& G_{1}\left(\bar{u}_{\mathrm{i}}^{C}, \bar{u}_{\mathrm{i}}^{B} ; \bar{v}_{\mathrm{ii}}^{C}, \bar{v}_{\mathrm{ii}}^{B}\right)=\sum_{\substack{\bar{u}_{\mathrm{i}}^{C, B} \Rightarrow\left\{\bar{u}_{1}^{C, B}, \bar{u}_{4}^{C, B}\right\} \\
\bar{v}_{\mathrm{ii}}^{C, B} \Rightarrow\left\{\bar{v}_{2}^{C, B}, \bar{v}_{3}^{C, B}\right\}}} f\left(\bar{u}_{4}^{C}, \bar{u}_{1}^{C}\right) f\left(\bar{u}_{1}^{B}, \bar{u}_{4}^{B}\right) f\left(\bar{v}_{2}^{C}, \bar{v}_{3}^{C}\right) f\left(\bar{v}_{3}^{B}, \bar{v}_{2}^{B}\right) \\
& \times f\left(\bar{v}_{3}^{C}, \bar{u}_{4}^{C}\right) f\left(\bar{v}_{2}^{B}, \bar{u}_{1}^{B}\right) Z_{a_{4}, b_{2}}\left(\bar{u}_{4}^{C} ; \bar{u}_{4}^{B} \mid \bar{v}_{2}^{C} ; \bar{v}_{2}^{B}\right) Z_{a_{1}, b_{3}}\left(\bar{u}_{1}^{B} ; \bar{u}_{1}^{C} \mid \bar{v}_{3}^{B} ; \bar{v}_{3}^{C}\right),
\end{aligned}
$$

and

$$
G_{2}\left(\bar{u}_{\mathrm{ii}}^{C}, \bar{u}_{\mathrm{ii}}^{B} ; \bar{v}_{\mathrm{i}}^{C}, \bar{v}_{\mathrm{i}}^{B}\right)=\sum_{\substack{\bar{u}_{\mathrm{i}}^{C, B} \Rightarrow\left\{\bar{u}_{2}^{C, B}, \bar{u}_{3}^{C, B}\right\} \\ \\ \bar{v}_{\mathrm{i}}^{C, B} \Rightarrow\left\{\bar{v}_{1}^{C, B}, \bar{v}_{4}^{C, B}\right\}}} e^{\beta_{1} \ell_{1}[0]+\beta_{3} \ell_{3}[0]+\left(\beta_{2}-\beta_{1}\right)\left(a-a_{2}\right)+\left(\beta_{2}-\beta_{3}\right) b_{4}}
$$




$$
\begin{aligned}
& \times f\left(\bar{u}_{3}^{C}, \bar{u}_{2}^{C}\right) f\left(\bar{u}_{2}^{B}, \bar{u}_{3}^{B}\right) f\left(\bar{v}_{1}^{C}, \bar{v}_{4}^{C}\right) f\left(\bar{v}_{4}^{B}, \bar{v}_{1}^{B}\right) f\left(\bar{v}_{4}^{C}, \bar{u}_{3}^{C}\right) f\left(\bar{v}_{1}^{B}, \bar{u}_{2}^{B}\right) \\
& \times Z_{a_{3}, b_{1}}\left(\bar{u}_{3}^{C} ; \bar{u}_{3}^{B} \mid \bar{v}_{1}^{C} ; \bar{v}_{1}^{B}\right) Z_{a_{2}, b_{4}}\left(\bar{u}_{2}^{B} ; \bar{u}_{2}^{C} \mid \bar{v}_{4}^{B} ; \bar{v}_{4}^{C}\right) .
\end{aligned}
$$

It is not difficult to see that the sum over partitions in (5.9) coincides with the sum in (2.8) up to relabeling of the subsets: $\bar{u}_{1}^{C, B} \rightarrow \bar{u}_{\mathrm{I}}^{C, B}, \bar{u}_{4}^{C, B} \rightarrow \bar{u}_{\mathrm{I}}^{C, B}, \bar{v}_{2}^{C, B} \rightarrow \bar{v}_{\mathrm{I}}^{C, B}$, and $\bar{v}_{3}^{C, B} \rightarrow \bar{v}_{\mathrm{II}}^{C, B}$. Thus, we conclude that $G_{1}=0$ unless $\bar{u}_{\mathrm{i}}^{C, B}=\varnothing$ and $\bar{v}_{\mathrm{ii}}^{C, B}=\varnothing$. Hence, $\bar{u}_{\mathrm{ii}}^{C, B}=\bar{u}^{C, B}, \bar{v}_{\mathrm{i}}^{C, B}=\bar{v}^{C, B}$, and $a_{1}=a_{4}=0, b_{2}=b_{3}=0$. Then due to $(2.9) G_{1}=1$.

Looking now at (5.10) and comparing it with (2.7) we see that they coincide up to the common prefactor $e^{\beta_{1} \ell_{1}[0]+\beta_{3} \ell_{3}[0]}$ and relabeling of the subsets: $\bar{u}_{2}^{C, B} \rightarrow \bar{u}_{\mathrm{I}}^{C, B}, \bar{u}_{3}^{C, B} \rightarrow \bar{u}_{\mathrm{I}}^{C, B}, \bar{v}_{1}^{C, B} \rightarrow \bar{v}_{\mathrm{I}}^{C, B}$, and $\bar{v}_{4}^{C, B} \rightarrow \bar{v}_{\mathrm{II}}^{C, B}$. Hence,

$$
G_{2}=e^{\beta_{1} \ell_{1}[0]+\beta_{3} \ell_{3}[0]} \mathcal{S}_{a, b}^{(\bar{\kappa})} .
$$

Substituting this into (5.8) and setting there $\bar{u}_{\mathrm{i}}^{C, B}=\varnothing$ and $\bar{v}_{\mathrm{ii}}^{C, B}=\varnothing$ we immediately arrive at (5.2).

\section{Form factor for off-diagonal partial zero modes}

Now we study the form factors of off-diagonal partial zero modes. We apply a strategy similar to the one used in [27], using the commutation relations of the zero modes and properties of the type (3.9), (3.10).

First, we note that the RTT-relation (1.1) implies in particular for the (partial) zero modes

$$
\begin{array}{llll}
{\left[T_{i i}[0], T_{j i}[0]\right]=T_{j i}[0],} & {\left[T_{i i}^{(l)}[0], T_{j i}^{(l)}[0]\right]=T_{j i}^{(l)}[0],} & i \neq j, & \\
{\left[T_{i j}[0], T_{i i}[0]\right]=T_{i j}[0],} & {\left[T_{i j}^{(l)}[0], T_{i i}^{(l)}[0]\right]=T_{i j}^{(l)}[0],} & i \neq j, \\
{\left[T_{i j}[0], T_{k i}[0]\right]=T_{k j}[0],} & {\left[T_{i j}^{(l)}[0], T_{k i}^{(l)}[0]\right]=T_{k j}^{(l)}[0],} & i \neq j \neq k, \quad l=1,2 .
\end{array}
$$

Now, using $T_{k l}[0]=T_{k l}^{(1)}[0]+T_{k l}^{(2)}[0]$ and $\left[T_{i j}^{(1)}[0], T_{k l}^{(2)}[0]\right]=0$ we conclude

$$
\begin{aligned}
& {\left[T_{i i}^{(1)}[0], T_{j i}[0]\right]=T_{j i}^{(l)}[0], \quad i \neq j,} \\
& {\left[T_{i j}[0], T_{i i}^{(1)}[0]\right]=T_{i j}^{(1)}[0], \quad i \neq j,} \\
& {\left[T_{i j}[0], T_{k i}^{(1)}[0]\right]=T_{k j}^{(1)}[0], \quad i \neq j \neq k,}
\end{aligned}
$$

which are the central relations that we will use for our calculations.

As a notation, we will note the form factor of $T_{i j}^{(1)}[0]$ as

$$
M_{a, b}^{(i, j)}\left(\bar{u}^{C}, \bar{v}^{C} ; \bar{u}^{B}, \bar{v}^{B}\right)=\mathbb{C}_{a^{\prime}, b^{\prime}}\left(\bar{u}^{C} ; \bar{v}^{C}\right) T_{i j}^{(1)}[0] \mathbb{B}_{a, b}\left(\bar{u}^{B} ; \bar{v}^{B}\right), \quad i, j=1,2,3,
$$

with $a^{\prime}=a+\delta_{i 1}-\delta_{j 1}$ and $b^{\prime}=b+\delta_{j 3}-\delta_{i 3}$, and where both vectors are on-shell.

\subsection{Form factor of $T_{12}^{(1)}[0]$}

We start with $M_{a, b}^{(1,2)}$, the form factor of $T_{12}^{(1)}[0]$. We make the calculation in details, the other ones following the same steps. To get $M_{a, b}^{(1,2)}$, we start with the form factor $M_{a+1, b}^{(2,2)}$ and send one of the parameters in $\bar{u}^{B}$ to infinity (keeping all the other parameters finite), using relations (3.8):

$$
\begin{aligned}
\lim _{w \rightarrow \infty} \frac{w}{c} M_{a+1, b}^{(2,2)}\left(\bar{u}^{C}, \bar{v}^{C} ;\left\{\bar{u}^{B}, w\right\}, \bar{v}^{B}\right) & =\lim _{w \rightarrow \infty} \frac{w}{c} \mathbb{C}_{a+1, b}\left(\bar{u}^{C} ; \bar{v}^{C}\right) T_{22}^{(1)}[0] \mathbb{B}_{a+1, b}\left(\left\{\bar{u}^{B}, w\right\} ; \bar{v}^{B}\right) \\
& =\mathbb{C}_{a+1, b}\left(\bar{u}^{C} ; \bar{v}^{C}\right) T_{22}^{(1)}[0] T_{12}[0] \mathbb{B}_{a, b}\left(\bar{u}^{B} ; \bar{v}^{B}\right) .
\end{aligned}
$$


Now, since $\mathbb{C}_{a+1, b}\left(\bar{u}^{C} ; \bar{v}^{C}\right)$ is on-shell with all parameters finite, it is annihilated by $T_{12}[0]$ due to (3.11). Thus, we can replace the product $T_{22}^{(1)}[0] T_{12}[0]$ by its commutator which in turn gives $T_{12}^{(1)}[0]$ through (6.1). It leads to

$$
\lim _{w \rightarrow \infty} \frac{w}{c} M_{a+1, b}^{(2,2)}\left(\bar{u}^{C}, \bar{v}^{C} ;\left\{\bar{u}^{B}, w\right\}, \bar{v}^{B}\right)=M_{a, b}^{(1,2)}\left(\bar{u}^{C}, \bar{v}^{C} ; \bar{u}^{B}, \bar{v}^{B}\right) .
$$

It remains to compute the limit of the explicit expression (4.1) for $M_{a+1, b}^{(2,2)}$, that was proved in the previous section. It is obvious that $\lim _{w \rightarrow \infty} \ell_{k}(w)=1, k=1,3$, and it has been shown in [27] that $^{5}$

$$
\lim _{w \rightarrow \infty} \frac{w}{c} \mathfrak{F}_{a+1, b}^{(2,2)}\left(\bar{u}^{C}, \bar{v}^{C} ;\left\{\bar{u}^{B}, w\right\}, \bar{v}^{B}\right)=\mathfrak{F}_{a, b}^{(1,2)}\left(\bar{u}^{C}, \bar{v}^{C} ; \bar{u}^{B}, \bar{v}^{B}\right) .
$$

Hence, we get expression (4.1) for $M_{a, b}^{(1,2)}$.

\subsection{Other form factors}

As already mentioned, the calculation for other form factors follows the same steps, so we just sketch the proofs.

Form factor of $\boldsymbol{T}_{\mathbf{2 3}}^{(\mathbf{1})}[\mathbf{0}]$. To get $M_{a, b}^{(2,3)}$, we start with $M_{a, b+1}^{(2,2)}\left(\bar{u}^{C}, \bar{v}^{C} ; \bar{u}^{B},\left\{\bar{v}^{B}, w\right\}\right)$ and take the limit $w \rightarrow \infty$. It makes appear $T_{23}[0]$ that annihilates $\mathbb{C}_{a, b+1}\left(\bar{u}^{C} ; \bar{v}^{C}\right)$. It leads to

$$
\lim _{w \rightarrow \infty} \frac{w}{c} M_{a, b+1}^{(2,2)}\left(\bar{u}^{C}, \bar{v}^{C} ; \bar{u}^{B},\left\{\bar{v}^{B}, w\right\}\right)=-M_{a, b}^{(2,3)}\left(\bar{u}^{C}, \bar{v}^{C} ; \bar{u}^{B}, \bar{v}^{B}\right) .
$$

Form factor of $\boldsymbol{T}_{\mathbf{2 1}}^{(\mathbf{1})}[\mathbf{0}]$. To get $M_{a, b}^{(2,1)}$, we start with $M_{a, b}^{(2,2)}\left(\left\{\bar{u}^{C}, w\right\}, \bar{v}^{C} ; \bar{u}^{B}, \bar{v}^{B}\right)$ and take the limit $w \rightarrow \infty$. It makes appear $T_{21}[0]$ on the left, and it annihilates $\mathbb{B}_{a, b}\left(\bar{u}^{C} ; \bar{v}^{C}\right)$ due to (3.11). Then, we can again replace the product $T_{21}[0] T_{22}^{(1)}[0]$ by its commutator, and we obtain

$$
\lim _{w \rightarrow \infty} \frac{w}{c} M_{a, b}^{(2,2)}\left(\left\{\bar{u}^{C}, w\right\}, \bar{v}^{C} ; \bar{u}^{B}, \bar{v}^{B}\right)=M_{a, b}^{(2,1)}\left(\bar{u}^{C}, \bar{v}^{C} ; \bar{u}^{B}, \bar{v}^{B}\right) .
$$

Form factor of $\boldsymbol{T}_{\mathbf{3 2}}^{(\mathbf{1})}[\mathbf{0}]$. The form factor $M_{a, b}^{(3,2)}$ is obtained through the limit

$$
\lim _{w \rightarrow \infty} \frac{w}{c} M_{a, b}^{(2,2)}\left(\bar{u}^{C},\left\{\bar{v}^{C}, w\right\} ; \bar{u}^{B}, \bar{v}^{B}\right)=-M_{a, b}^{(3,2)}\left(\bar{u}^{C}, \bar{v}^{C} ; \bar{u}^{B}, \bar{v}^{B}\right) .
$$

Form factor of $\boldsymbol{T}_{\mathbf{1 3}}^{(\mathbf{1})}[\mathbf{0}]$. To get $M_{a, b}^{(1,3)}$, it is convenient to start with the already known form factor $M_{a+1, b}^{(2,3)}\left(\bar{u}^{C}, \bar{v}^{C} ;\left\{\bar{u}^{B}, w\right\}, \bar{v}^{B}\right)$. Taking the limit $w \rightarrow \infty$ we obtain $T_{12}[0]$ that annihilates $\mathbb{C}_{a+1, b}\left(\bar{u}^{C} ; \bar{v}^{C}\right)$. Hence, due to the last equation (6.1) we obtain

$$
\lim _{w \rightarrow \infty} \frac{w}{c} M_{a+1, b}^{(2,3)}\left(\bar{u}^{C}, \bar{v}^{C} ;\left\{\bar{u}^{B}, w\right\}, \bar{v}^{B}\right)=M_{a, b}^{(1,3)}\left(\bar{u}^{C}, \bar{v}^{C} ; \bar{u}^{B}, \bar{v}^{B}\right) .
$$

Form factor of $\boldsymbol{T}_{\mathbf{3 1}}^{(\mathbf{1})}[\mathbf{0}]$. To get $M_{a, b}^{(3,1)}$, we start with $M_{a, b}^{(3,2)}\left(\left\{\bar{u}^{C}, w\right\}, \bar{v}^{C} ; \bar{u}^{B}, \bar{v}^{B}\right)$ and take the limit $w \rightarrow \infty$. We obtain

$$
\lim _{w \rightarrow \infty} \frac{w}{c} M_{a, b}^{(3,2)}\left(\left\{\bar{u}^{C}, w\right\}, \bar{v}^{C} ; \bar{u}^{B}, \bar{v}^{B}\right)=M_{a, b}^{(3,1)}\left(\bar{u}^{C}, \bar{v}^{C} ; \bar{u}^{B}, \bar{v}^{B}\right) .
$$

Thus, starting from one initial form factor $M_{a, b}^{(2,2)}$ we can obtain all other form factors $M_{a, b}^{(i, j)}$ in the special limits of the Bethe parameters. This property is a direct consequence of the property of the form factors of the monodromy matrix entries $\mathcal{F}_{a, b}^{(i, j)}[30]$.

\footnotetext{
${ }^{5}$ Strictly speaking the proof of [28] was done for the full form factor, but it extends straightforwardly to the universal form factor.
} 
Consistency with morphisms. It is worth mentioning that there exist also other relations between different form factors $M_{a, b}^{(i, j)}[28,30]$. These relations appear due to morphisms of the algebra (1.1). It was shown in [3] that the mappings

$$
\psi: T_{i j}(u) \mapsto T_{j i}(u), \quad \varphi: T_{i j}(u) \mapsto T_{4-j, 4-i}(-u),
$$

define morphisms of the algebra (1.1). Hereby, the mapping $\varphi$ is an isomorphism, while $\psi$ is an antimorphism. Both mappings (6.9) exchange the partial monodromy matrices of the composite model [29]

$$
\psi: T_{i j}^{(l)}(u) \mapsto T_{j i}^{(3-l)}(u), \quad \varphi: T_{i j}^{(l)}(u) \mapsto T_{4-j, 4-i}^{(3-l)}(-u) .
$$

Transformations (6.9) induce relations between different form factors of the monodromy matrix entries $\mathcal{F}_{a, b}^{(i, j)}$ (see $[27,28]$ for details). The latest, in their turn, give us relations between the universal form factors $\mathfrak{F}_{a, b}^{(i, j)}$

$$
\begin{aligned}
& \mathfrak{F}_{a, b}^{(i, j)}\left(\bar{u}^{C}, \bar{v}^{C} ; \bar{u}^{B}, \bar{v}^{B}\right)=-\mathfrak{F}_{a^{\prime}, b^{\prime}}^{(j, i)}\left(\bar{u}^{B}, \bar{v}^{B} ; \bar{u}^{C}, \bar{v}^{C}\right), \\
& \mathfrak{F}_{a, b}^{(i, j)}\left(\bar{u}^{C}, \bar{v}^{C} ; \bar{u}^{B}, \bar{v}^{B}\right)=\mathfrak{F}_{b, a}^{(4-j, 4-i)}\left(-\bar{v}^{C},-\bar{u}^{C} ;-\bar{v}^{B},-\bar{u}^{B}\right) .
\end{aligned}
$$

Thus, actually it is enough to compute only four form factors of the partial zero modes $T_{1 j}^{(1)}[0]$ $(j=1,2,3)$ and $T_{22}^{(1)}[0]$. All other form factors can be obtained by the mappings described above. Using the explicit determinant representations for the from factors one can check that they enjoy both the limiting procedures (6.2), (6.4)-(6.8), and the transformations (6.10).

Generalisation to $\mathbf{G L}(\boldsymbol{N})$. One can apply the same procedure to the $\mathrm{GL}(N)$ case. In particular, the commutation relations (6.1) are still valid, and the limits of the type (3.9), (3.10) and (6.3) have been proven for GL $(N)$ in [27]. The singular vector properties of the type (3.11) were obtained for $\mathrm{GL}(N)$ in [26]. All that allows us to relate the different form factors in the same way we did for GL(3). It is easy to check that the Conjecture (4.3) is consistent with these relations.

\section{Conclusion}

In this paper we have studied form factors of the partial zero modes in a composite generalized model with GL(3)-invariant $R$-matrix. We have reduced these form factors to the ones of the monodromy matrix entries $T_{i j}(u)$ considered in our previous publications. As we have mentioned already, it means that we have a solution of the inverse scattering problem in the weak sense. Apparently the same type of the formulas remains true for GL( $N)$-invariant models with $N>3$.

We are planning to apply these results to the study of form factors of local operators in the model of one-dimensional two-component Bose gas with $\delta$-function interaction [35, 39]. Several form factors in this model were studied already in the framework of the coordinate Bethe ansatz for some particular cases of Bethe vectors [31]. We are going to apply the algebraic Bethe ansatz techniques in order to compute the form factors in the general case. This model possesses GL(3)invariant $R$-matrix (1.2) and can be considered in the framework of the scheme described in the present paper. However the asymptotic expansion of the monodromy matrices (1.6) in the case of Bose gas should be modified. In its turn, this modification leads to a modification of the partial zero modes. Nevertheless the derivation of determinant representations for the form factors of the diagonal partial zero modes $T_{i i}^{(1)}[0]$ does not change. A possibility to use this result for obtaining all other form factors is not obvious, however they can be calculated by a straightforward method similar to the one that we used for the calculation of $T_{i i}^{(1)}[0]$ in the present paper. This will be the subject of our further publication. 


\section{Acknowledgements}

The work of S.P. was supported in part by RFBR-Ukraine grant 14-01-90405-ukr-a. N.A.S. was supported by the Program of RAS "Nonlinear Dynamics in Mathematics and Physics", RFBR14-01-00860-a, RFBR-13-01-12405-ofi-m2.

\section{References}

[1] Belliard S., Pakuliak S., Ragoucy E., Slavnov N.A., Highest coefficient of scalar products in SU(3)-invariant integrable models, J. Stat. Mech. Theory Exp. 2012 (2012), P09003, 17 pages, arXiv:1206.4931.

[2] Belliard S., Pakuliak S., Ragoucy E., Slavnov N.A., The algebraic Bethe ansatz for scalar products in SU(3)invariant integrable models, J. Stat. Mech. Theory Exp. 2012 (2012), P10017, 25 pages, arXiv:1207.0956.

[3] Belliard S., Pakuliak S., Ragoucy E., Slavnov N.A., Bethe vectors of GL(3)-invariant integrable models, J. Stat. Mech. Theory Exp. 2013 (2013), P02020, 24 pages, arXiv:1210.0768.

[4] Belliard S., Pakuliak S., Ragoucy E., Slavnov N.A., Form factors in SU(3)-invariant integrable models, J. Stat. Mech. Theory Exp. 2013 (2013), P04033, 16 pages, arXiv:1211.3968.

[5] Caux J.-S., Maillet J.M., Computation of dynamical correlation functions of Heisenberg chains in a magnetic field, Phys. Rev. Lett. 95 (2005), 077201, 3 pages, cond-mat/0502365.

[6] Enriquez B., Khoroshkin S., Pakuliak S., Weight functions and Drinfeld currents, Comm. Math. Phys. 276 (2007), 691-725, math.QA/0610398.

[7] Faddeev L.D., How the algebraic Bethe ansatz works for integrable models, in Symétries Quantiques (Les Houches, 1995), North-Holland, Amsterdam, 1998, 149-219, hep-th/9605187.

[8] Göhmann F., Klümper A., Seel A., Integral representations for correlation functions of the $X X Z$ chain at finite temperature, J. Phys. A: Math. Gen. 37 (2004), 7625-7651, hep-th/0405089.

[9] Göhmann F., Klümper A., Seel A., Integral representation of the density matrix of the $X X Z$ chain at finite temperatures, J. Phys. A: Math. Gen. 38 (2005), 1833-1841, cond-mat/0412062.

[10] Izergin A.G., Korepin V.E., The quantum inverse scattering method approach to correlation functions, Comm. Math. Phys. 94 (1984), 67-92.

[11] Izergin A.G., Korepin V.E., Reshetikhin N.Yu., Correlation functions in a one-dimensional Bose gas, J. Phys. A: Math. Gen. 20 (1987), 4799-4822.

[12] Khoroshkin S., Pakuliak S., A computation of universal weight function for quantum affine algebra $U_{q}\left(\widehat{\mathfrak{g l}}_{N}\right)$, J. Math. Kyoto Univ. 48 (2008), 277-321, arXiv:0711.2819.

[13] Khoroshkin S., Pakuliak S., Tarasov V., Off-shell Bethe vectors and Drinfeld currents, J. Geom. Phys. 57 (2007), 1713-1732, math.QA/0610517.

[14] Kitanine N., Kozlowski K., Maillet J.M., Slavnov N.A., Terras V., On correlation functions of integrable models associated with the six-vertex R-matrix, J. Stat. Mech. Theory Exp. 2007 (2007), P01022, 17 pages, hep-th/0611142.

[15] Kitanine N., Kozlowski K., Maillet J.M., Slavnov N.A., Terras V., Algebraic Bethe ansatz approach to the asymptotic behavior of correlation functions, J. Stat. Mech. Theory Exp. 2009 (2009), P04003, 66 pages, arXiv:0808.0227.

[16] Kitanine N., Kozlowski K., Maillet J.M., Slavnov N.A., Terras V., On the thermodynamic limit of form factors in the massless $X X Z$ Heisenberg chain, J. Math. Phys. 50 (2009), 095209, 24 pages, arXiv:0903.2916.

[17] Kitanine N., Kozlowski K., Maillet J.M., Slavnov N.A., Terras V., A form factor approach to the asymptotic behavior of correlation functions, J. Stat. Mech. Theory Exp. 2011 (2011), P12010, 27 pages, arXiv:1110.0803.

[18] Kitanine N., Maillet J.M., Slavnov N.A., Terras V., Spin-spin correlation functions of the $X X Z$ - $\frac{1}{2}$ Heisenberg chain in a magnetic field, Nuclear Phys. B 641 (2002), 487-518, hep-th/0201045.

[19] Kitanine N., Maillet J.M., Slavnov N.A., Terras V., Master equation for spin-spin correlation functions of the $X X Z$ chain, Nuclear Phys. B 712 (2005), 600-622, hep-th/0406190.

[20] Kitanine N., Maillet J.M., Terras V., Form factors of the $X X Z$ Heisenberg spin- $\frac{1}{2}$ finite chain, Nuclear Phys. B 554 (1999), 647-678, math-ph/9807020.

[21] Korepin V.E., Correlation functions of the one-dimensional Bose gas in the repulsive case, Comm. Math. Phys. 94 (1984), 93-113. 
[22] Korepin V.E., Bogoliubov N.M., Izergin A.G., Quantum inverse scattering method and correlation functions, Cambridge Monographs on Mathematical Physics, Cambridge University Press, Cambridge, 1993.

[23] Kulish P.P., Reshetikhin N.Yu., Diagonalisation of $\operatorname{GL}(N)$ invariant transfer matrices and quantum $N$-wave system (Lee model), J. Phys. A: Math. Gen. 16 (1983), L591-L596.

[24] Kulish P.P., Reshetikhin N.Yu., GL3-invariant solutions of the Yang-Baxter equation and associated quantum systems, J. Sov. Math. 34 (1986), 1948-1971.

[25] Maillet J.M., Terras V., On the quantum inverse scattering problem, Nuclear Phys. B 575 (2000), 627-644, hep-th/9911030.

[26] Mukhin E., Tarasov V., Varchenko A., Bethe eigenvectors of higher transfer matrices, J. Stat. Mech. Theory Exp. 2006 (2006), P08002, 44 pages, math.QA/0605015.

[27] Pakuliak S., Ragoucy E., Slavnov N.A., Determinant representations for form factors in quantum integrable models with the GL(3)-invariant R-matrix, Theoret. and Math. Phys. 181 (2014), 1566-1584, arXiv:1406.5125.

[28] Pakuliak S., Ragoucy E., Slavnov N.A., Form factors in quantum integrable models with GL(3)-invariant R-matrix, Nuclear Phys. B 881 (2014), 343-368, arXiv:1312.1488.

[29] Pakuliak S., Ragoucy E., Slavnov N.A., GL(3)-based quantum integrable composite models. I. Bethe vectors, SIGMA 11 (2015), 063, 20 pages, arXiv:1501.07566.

[30] Pakuliak S., Ragoucy E., Slavnov N.A., Zero modes method and form factors in quantum integrable models, Nuclear Phys. B 893 (2015), 459-481, arXiv:1412.6037.

[31] Pozsgay B., van Gerven Oei W.V., Kormos M., On form factors in nested Bethe Ansatz systems, J. Phys. A: Math. Theor. 45 (2012), 465007, 34 pages, arXiv:1204.4037.

[32] Reshetikhin N.Yu., Calculation of the norm of Bethe vectors in models with SU(3) symmetry, J. Sov. Math. 46 (1989), 1694-1706.

[33] Sklyanin E.K., Takhtadzhyan L.A., Faddeev L.D., Quantum inverse problem method. I, Theoret. and Math. Phys. 40 (1979), 688-706.

[34] Slavnov N.A., The algebraic Bethe ansatz and quantum integrable systems, Russian Math. Surveys 62 (2007), 727-766.

[35] Sutherland B., Further results for the many-body problem in one dimension, Phys. Rev. Lett. 20 (1968), 98-100.

[36] Takhtadzhan L.A., Faddeev L.D., The quantum method for the inverse problem and the Heisenberg $X Y Z$ model, Russian Math. Surveys 34 (1979), no. 5, 11-68.

[37] Varchenko A.N., Tarasov V.O., Jackson integral representations for solutions of the Knizhnik-Zamolodchikov quantum equation, St. Petersburg Math. J. 6 (1994), 275-313, hep-th/9311040.

[38] Wheeler M., Scalar products in generalized models with SU(3)-symmetry, Comm. Math. Phys. 327 (2014), 737-777, arXiv:1204.2089.

[39] Yang C.N., Some exact results for the many-body problem in one dimension with repulsive delta-function interaction, Phys. Rev. Lett. 19 (1967), 1312-1315. 